DESY 05-008

hep-ph/0605310

SFB-CPP-06/25

May 2006

\title{
Target mass and finite momentum transfer corrections to unpolarized and polarized diffractive scattering
}

\author{
Johannes Blümlein ${ }^{a}$, Bodo Geyer ${ }^{b}$ and Dieter Robaschik ${ }^{a, c}$ \\ a Deutsches Elektronen-Synchrotron, DESY, \\ Platanenallee 6, D-15738 Zeuthen, Germany \\ ${ }^{b}$ Center for Theoretical Studies and Institute of Theoretical Physics, \\ Leipzig University, Augustusplatz 10, D-04109 Leipzig, Germany \\ ${ }^{c}$ Brandenburgische Technische Universität Cottbus, Fakultät 1, \\ PF 101344, D-03013 Cottbus, Germany
}

\begin{abstract}
A quantum field theoretic treatment of inclusive deep-inelastic diffractive scattering is given. The process can be described in the general framework of non-forward scattering processes using the light-cone expansion in the generalized Bjorken region. Target mass and finite $t$ corrections of the diffractive hadronic tensor are derived at the level of the twist-2 contributions both for the unpolarized and the polarized case. They modify the expressions contributing in the limit $t, M^{2} \rightarrow 0$ for larger values of $\beta$ or/and $t$ in the region of low $Q^{2}$. The different diffractive structure functions are expressed through integrals over the relative momentum of non-perturbative $t$-dependent 2 -particle distribution functions. In the limit $t, M^{2} \rightarrow 0$ these distribution functions are the diffractive parton distribution. Relations between the different diffractive structure functions are derived.
\end{abstract}

PACS: 24.85.+p, 13.88.+e, 11.30.Cp

Keywords: Diffractive Scattering, Target Mass Effects, Finite momentum transfer corrections, Twist decomposition, Nonlocal light-cone operators, Multivalued distribution amplitude, Generalized Bjorken limit. 


\section{Introduction}

Deep inelastic diffractive lepton-nucleon scattering was observed at the electron-proton collider HERA some years ago [1]. This process is measured in detail by now [2] and the structure function $F_{2}^{D}\left(x, Q^{2}\right)$ was extracted. ${ }^{1}$ The experimental measurements clearly showed that the scaling violations of the deep-inelastic and the diffractive structure functions in the deep-inelastic regime, after an appropriate change of kinematic variables, are the same. Furthermore the ratio of the two quantities, did not vary strongly, cf. [4]. While the former property is clearly of perturbative nature, the latter is of non-perturbative origin.

The process of deep-inelastic diffractive scattering was first described phenomenologically [5]. Diffractive events are characterized by a rapidity gap between the diffractive nucleon and the remaining part of the produced hadrons, which is sufficiently large. Actually it is this experimental signature along with factorization for the twist-2 contributions [6] for this process, which allows to give a consistent field theoretic description. Due to this phenomenological considerations containing reference to specific pomeron models can be thoroughly avoided. In the limit of vanishing target masses the scattering cross sections and relations between the diffractive structure functions were derived in Refs. [7-9] for unpolarized and polarized diffractive scattering. In [7] we showed, that the scaling violations in the deep-inelastic and deeply inelastic diffractive case have to be the same due to the fact that the scaling violations are actually those of the operators which remain taking the respective matrix elements confirming the experimental observation. The set of structure functions which emerge in both scattering cross sections is actually larger than measured in current experiments. At low scales of $Q^{2}$ target mass effects become relevant similar to the case of deep-inelastic scattering [10-14], see also [15].

In the present paper we extend the picture developed in the massless case $[7,8]$ to the case of finite target masses and finite values of $t$ on the level of twist-2 operators in the light cone expansion [16]. While in absence of mass effects the two-particle problem could effectively be reduced to a single particle description for the case $t \rightarrow 0$, this is no longer the case for finite values of $t$ and/or target masses. Here two-particle effects become relevant, which do not allow for a direct partonic description. The variables $t=\left(p_{i}-p_{f}\right)^{2}$ and $M^{2}=p_{i}^{2}=p_{f}^{2}$ are closely connected and the simplification emerges if these scales vanish. Yet one may still follow the field theoretic picture developed in $[7,8]$ in the general case $M^{2}, t \neq 0$ and derive expressions for the diffractive structure functions including relations between them. At low scales $Q^{2}$ and large values of $\beta$ target mass corrections have to be considered in the experimental analysis. This generally applies also to finite values of $t$, unless the scale $Q^{2}$ is large enough. The diffractive structure functions are found as integrals over two-particle correlation functions $f\left(z_{+}, z_{-} ; t\right)$ between the incoming and outgoing nucleon. Here, $z_{ \pm}$denote the corresponding collinear light-cone momentum fractions and $t$ is the relative momentum transfer squared between the incoming and outgoing proton momentum. We refer to the formalism of non-forward Compton scattering, cf. [17], and apply the general group theoretical algorithm of decomposing off-cone tensor operators into operators of definite geometric twist [18-20] to determine the contributions at twist 2. The analysis can be generalized to operators of higher twist. On the level of the various correlation functions relations can be established. These relations correspond to relations between structure functions, cf. $[12,21-24]$. In the case of deeply virtual Compton scattering relations of this type were found in [25-27] before.

The present field-theoretic formalism for deep-inelastic diffractive scattering was also devel-

\footnotetext{
${ }^{1}$ The measurement of the longitudinal diffractive structure function $F_{L}^{D}\left(x, Q^{2}\right)$ has not yet been possible, but would be important. For the DIS structure function cf. [3]. Likewise the polarized diffractive structure functions $g_{1,2}^{D}\left(x, Q^{2}\right)$ should be measured in the future to reveal the effects of nucleon polarization in this process.
} 
oped in view of possible future measurements of the respective operator-matrixelements using lattice techniques, as successfully applied in case of the moments of the deep-inelastic structure functions [28]. We regard it as a challenge in future investigations to verify the experimentally observed ratio of moments in the diffractive and deep-inelastic case with these technologies. This ratio still awaits a rigorous non-perturbative explanation.

The paper is organized as follows. In section 2 we describe the basic formalism. In section 3 the symmetric part of the Compton amplitude is dealt with, through which the diffractive structure functions for unpolarized nucleons are derived. The polarized structure functions are determined in section 4 . In section 5 we derive relations between different structure functions and section 6 contains the conclusions.

\section{Basic Formalism}

In order to compute the twist-2 target mass and finite momentum transfer corrections in polarized and unpolarized deep inelastic diffractive scattering we briefly recall the notations and conventions used in previous papers $[7,8]$ by two of the present authors. The process of deepinelastic diffractive scattering belongs to the class of semi-inclusive processes and is described by the diagram in Figure 1. The differential scattering cross section for single-photon exchange is given by

$$
\mathrm{d}^{5} \sigma_{\text {diffr }}=\frac{1}{2\left(s-M^{2}\right)} \frac{1}{4} d P S^{(3)} \sum_{\text {spins }} \frac{e^{4}}{Q^{2}} L_{\mu \nu} W^{\mu \nu} .
$$

Here $s=\left(p_{1}+l\right)^{2}$ is the cms energy squared of the process and $M$ denotes the nucleon mass. The phase space $d P S^{(3)}$ depends on five variables since one final state mass varies. We choose as basic variables

$$
x=\frac{Q^{2}}{Q^{2}+W^{2}-M^{2}}=-\frac{q^{2}}{2 q p_{1}},
$$

the photon virtuality $Q^{2}=-q^{2}, t=\left(p_{2}-p_{1}\right)^{2}$ the 4 -momentum difference squared between incoming and outgoing nucleon, a variable describing the non-forwardness w.r.t. the incoming proton direction,

$$
x_{\mathbb{P}}=\frac{Q^{2}+M_{X}^{2}-t}{Q^{2}+W^{2}-M^{2}}=-\frac{q p_{-}}{q p_{1}} \geq x
$$

for $M_{X}^{2}>t$, and the angle $\Phi$ between the lepton plane $\boldsymbol{p}_{1} \times \boldsymbol{l}$ and the hadron plane $\boldsymbol{p}_{1} \times \boldsymbol{p}_{2}$,

$$
\cos \Phi=\frac{\left(\boldsymbol{p}_{1} \times \boldsymbol{l}\right) \cdot\left(\boldsymbol{p}_{1} \times \boldsymbol{p}_{2}\right)}{\left|\boldsymbol{p}_{1} \times \boldsymbol{l}\right|\left|\boldsymbol{p}_{1} \times \boldsymbol{p}_{2}\right|}
$$

where $p_{ \pm}=p_{2} \pm p_{1}, W^{2}=\left(p_{1}+q\right)^{2}$ denotes the hadronic mass squared and the diffractive mass squared is given by $M_{X}^{2}=\left(q-p_{-}\right)^{2}$.

The momenta $p_{ \pm}$obey

$$
\left(p_{+} p_{-}\right)=0, \quad \frac{p_{+}^{2}}{p_{-}^{2}}=\frac{4 M^{2}}{t}-1 .
$$

For later use we refer to the variables $\eta$ and $\beta$ defined by

$$
\eta=\frac{q p_{-}}{q p_{+}}=\frac{-x_{\mathbb{P}}}{2-x_{\mathbb{P}}} \in\left[-1, \frac{-x}{2-x}\right], \quad \beta=\frac{q^{2}}{2 q p_{-}}=\frac{x}{x_{\mathbb{P}}} \leq 1,
$$


as well as to the transverse momentum variable

$$
\pi_{-}=p_{+}-\frac{p_{-}}{\eta}, \quad\left(q \pi_{-}\right)=0
$$

The variables $x, x_{\mathbb{P}}, \beta$ and $\eta$ obey the inequalities

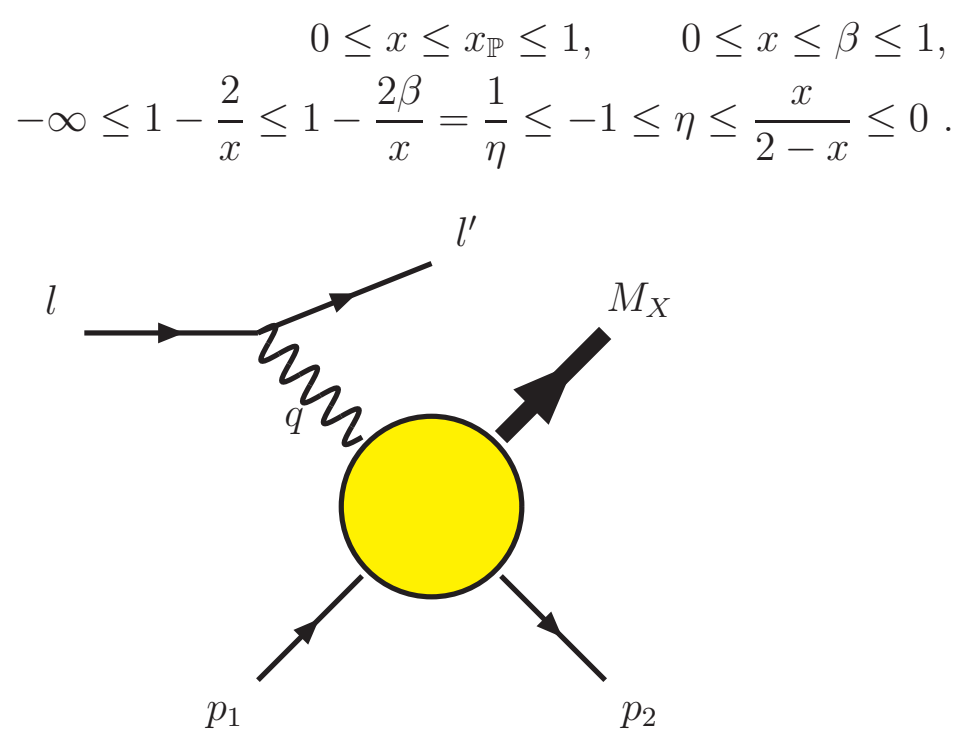

Figure 1: The virtual photon-hadron amplitude for diffractive ep scattering

For the spin averaged cross section, the leptonic tensor is symmetric. Taking into account conservation of the electromagnetic current one obtains [7]

$$
W_{\mu \nu}^{s}=-g_{\mu \nu}^{T} W_{1}^{s}+p_{1 \mu}^{T} p_{1 \nu}^{T} \frac{W_{2}^{s}}{M^{2}}+p_{2 \mu}^{T} p_{2 \nu}^{T} \frac{W_{3}^{s}}{M^{2}}+\left[p_{1 \mu}^{T} p_{2 \nu}^{T}+p_{2 \mu}^{T} p_{1 \nu}^{T}\right] \frac{W_{4}^{s}}{M^{2}} .
$$

Here and in the following we do not assume implicitly, that azimuthal integrals are performed as sometimes done in experiment. In the latter case the number of contributing structure function reduces.

In the case of polarized nucleons we consider the initial state spin-vector $S_{1} \equiv S, S^{2}=-M^{2}$, only and sum over the spin of the outgoing hadrons. One usually considers the longitudinal (||) and transverse $(\perp)$ spin projections choosing

$$
\begin{aligned}
S_{\|} & =(0 ; 0,0,0, M) \\
S_{\perp} & =(0 ; \cos \gamma, \sin \gamma, 0) M .
\end{aligned}
$$

Here $\gamma$ denotes the azimuthal angle. The antisymmetric part of the hadronic tensor was derived in [8] and is given by

$$
\begin{array}{rr}
W_{\mu \nu}^{a}= & i\left[p_{1 \mu}^{T} p_{2 \nu}^{T}-p_{1 \nu}^{T} p_{2 \mu}^{T}\right] \varepsilon_{p_{1} p_{2} q S} \frac{W_{1}^{a}}{M^{6}}+\quad i\left[p_{1 \mu}^{T} \varepsilon_{\nu S p_{1} q}-p_{1 \nu}^{T} \varepsilon_{\mu S p_{1} q}\right] \frac{W_{2}^{a}}{M^{4}} \\
+ & i\left[p_{2 \mu}^{T} \varepsilon_{\nu S p_{1} q}-p_{2 \nu}^{T} \varepsilon_{\mu S p_{1} q}\right] \frac{W_{3}^{a}}{M^{4}}+\quad i\left[p_{1 \mu}^{T} \varepsilon_{\nu S p_{2} q}-p_{1 \nu}^{T} \varepsilon_{\mu S p_{2} q}\right] \frac{W_{4}^{a}}{M^{4}} \\
+ & i\left[p_{2 \mu}^{T} \varepsilon_{\nu S p_{2} q}-p_{2 \nu}^{T} \varepsilon_{\mu S p_{2} q}\right] \frac{W_{5}^{a}}{M^{4}}+i\left[p_{1 \mu}^{T} \varepsilon_{\nu p_{1} p_{2} S}^{T}-p_{1 \nu}^{T} \varepsilon_{\mu p_{1} p_{2} S}^{T}\right] \frac{W_{6}^{a}}{M^{4}} \\
+\quad & i\left[p_{2 \mu}^{T} \varepsilon_{\nu p_{1} p_{2} S}^{T}-p_{2 \nu}^{T} \varepsilon_{\mu p_{1} p_{2} S}^{T}\right] \frac{W_{7}^{a}}{M^{4}}+
\end{array}
$$


We will specify below which terms of this general structures contribute in case of deep-inelastic diffractive scattering. The kinematic factors above are constructed out of the four-vectors $q, p_{1}, p_{2}$ and $S$ as well as $g_{\mu \nu}$ and $\varepsilon_{v_{0} v_{1} v_{2} v_{3}}$ using

$$
\begin{aligned}
p_{\mu}^{T} & =p_{\mu}-q_{\mu} \frac{q \cdot p}{q^{2}}, g_{\mu \nu}^{T}=g_{\mu \nu}-\frac{q_{\mu} q_{\nu}}{q^{2}} \\
\varepsilon_{\mu v_{1} v_{2} v_{3}}^{T} & =\varepsilon_{\mu v_{1} v_{2} v_{3}}-\varepsilon_{q v_{1} v_{2} v_{3}} \frac{q_{\mu}}{q^{2}} \\
\varepsilon_{\mu \nu v_{1} v_{2}}^{T T} & =\varepsilon_{\mu \nu v_{1} v_{2}}-\varepsilon_{q \nu v_{1} v_{2}} \frac{q_{\mu}}{q^{2}}-\varepsilon_{\mu q v_{1} v_{2}} \frac{q_{\nu}}{q^{2}} .
\end{aligned}
$$

At the level of the twist-2 contributions factorization holds for diffractive scattering [6]. A. Mueller's generalized optical theorem [29] allows to move the final state proton into an initial state anti-proton, where both particle momenta are separated by $t$ and form a 'quasi twoparticle' state. The correctness of this procedure within the light-cone expansion relies, first, on the rapidity gap between the outgoing proton and the remainder hadronic part with invariant mass $M_{X}$ and, second, on the special property of matrix elements of the light-cone operators which contain no absorptive part. The structure functions for the diffractive process can thus be obtained by analyzing the absorptive part of the expectation value

$$
T_{\mu \nu}(x)=\left\langle p_{1},-p_{2}, S ; t\left|\widehat{T}_{\mu \nu}(x)\right| p_{1},-p_{2}, S ; t\right\rangle,
$$

with $\widehat{T}_{\mu \nu}$ defined as

$$
\widehat{T}_{\mu \nu}(x) \equiv i R T\left[J_{\mu}\left(\frac{x}{2}\right) J_{\nu}\left(-\frac{x}{2}\right) \mathcal{S}\right]
$$

As shown in $[17,30]$ the operator $\widehat{T}_{\mu \nu}$ is represented in lowest order of the non-local light-cone expansion by

$$
\widehat{T}_{\mu \nu}(x) \approx-e^{2} \frac{\tilde{x}_{\lambda}}{2 \mathrm{i} \pi^{2}\left(x^{2}-i \epsilon\right)^{2}}\left[S_{\mu \nu \mid}^{\alpha \lambda} O_{\alpha}\left(\frac{\tilde{x}}{2},-\frac{\tilde{x}}{2}\right)-\epsilon_{\mu \nu}{ }^{\alpha \lambda} O_{5 \alpha}\left(\frac{\tilde{x}}{2},-\frac{\tilde{x}}{2}\right)\right],
$$

where

$$
S_{\mu \nu \mid \alpha \lambda}=g_{\mu \alpha} g_{\nu \lambda}+g_{\mu \lambda} g_{\nu \alpha}-g_{\mu \nu} g_{\alpha \lambda}
$$

$\tilde{x}$ denotes a light-like vector related to $x$,

$$
\tilde{x}=x-n\left[(n x)-\sqrt{(n x)^{2}-n^{2} x^{2}}\right]
$$

with $n$ a normalized time-like vector, $n^{2}=1$, and the bi-local light-ray operators $O_{\alpha}$ and $O_{\alpha}^{5}$ read

$$
\begin{aligned}
& O_{\alpha}\left(\kappa_{1} \tilde{x}, \kappa_{2} \tilde{x}\right)=i\left(\Omega_{\alpha}\left(\kappa_{1} \tilde{x}, \kappa_{2} \tilde{x}\right)-\Omega_{\alpha}\left(\kappa_{2} \tilde{x}, \kappa_{1} \tilde{x}\right)\right) \\
& O_{\alpha}^{5}\left(\kappa_{1} \tilde{x}, \kappa_{2} \tilde{x}\right)=\Omega_{\alpha}^{5}\left(\kappa_{1} \tilde{x}, \kappa_{2} \tilde{x}\right)+\Omega_{\alpha}^{5}\left(\kappa_{2} \tilde{x}, \kappa_{1} \tilde{x}\right)
\end{aligned}
$$

with

$$
\begin{aligned}
& \Omega_{\alpha}\left(\kappa_{1} \tilde{x}, \kappa_{2} \tilde{x}\right)=R T\left(: \bar{\psi}\left(\kappa_{1} \tilde{x}\right) \gamma_{\alpha} U\left(\kappa_{1} \tilde{x}, \kappa_{2} \tilde{x}\right) \psi\left(\kappa_{2} \tilde{x}\right): \mathcal{S}\right) \\
& \Omega_{\alpha}^{5}\left(\kappa_{1} \tilde{x}, \kappa_{2} \tilde{x}\right)=R T\left(: \bar{\psi}\left(\kappa_{1} \tilde{x}\right) \gamma^{5} \gamma_{\alpha} U\left(\kappa_{1} \tilde{x}, \kappa_{2} \tilde{x}\right) \psi\left(\kappa_{2} \tilde{x}\right): \mathcal{S}\right)
\end{aligned}
$$

where $\kappa_{1}=-\kappa_{2}=1 / 2$, cf. [17]. As is well-known, these operators contain contributions of up to twist four [18]. The scattering amplitude is obtained by the Fourier transform of the operator 
$\widehat{T}_{\{\mu \nu\}}(x)$ and forming the matrix element (2.17). Here we want to study its twist-2 contributions including the target mass and finite momentum transfer corrections. This is obtained by harmonic extension of the twist-2 light-cone operators $\Omega_{\alpha}^{\mathrm{tw} 2}\left(\kappa_{1} \tilde{x}, \kappa_{2} \tilde{x}\right)$ and $\Omega_{5 \alpha}^{\mathrm{tw} 2}\left(\kappa_{1} \tilde{x}, \kappa_{2} \tilde{x}\right)$ to twist-2 operators $\Omega_{\alpha}^{\mathrm{tw} 2}\left(\kappa_{1} x, \kappa_{2} x\right)$ and $\Omega_{5 \alpha}^{\mathrm{tw} 2}\left(\kappa_{1} x, \kappa_{2} x\right)$ defined off-cone. This procedure has been performed for QCD vector operators using group theoretical methods already in Ref. [31] and used for the target mass and $t$-corrections of virtual Compton scattering in Ref. [27], cf. also [32]. A general procedure for determining the complete (infinite) twist decomposition of off-cone vector operators was introduced in Ref. [19] and recently extended to arbitrary tensor operators in Ref. [20]. It should be emphasized that this method works at operator level before taking matrix elements. As a result the twist-2 Compton operator off the light-cone reads $[27,31]$

$$
\begin{aligned}
\widehat{T}_{\mu \nu}^{\mathrm{tw} 2}(q) & =\int \mathrm{d}^{4} x \mathrm{e}^{\mathrm{i} q x} \widehat{T}_{\mu \nu}^{\mathrm{tw} 2}(x) \\
& =-e^{2} \int \frac{d^{4} x}{2 \mathrm{i} \pi^{2}} \frac{\mathrm{e}^{\mathrm{i} q x} x_{\lambda}}{\left(x^{2}-\mathrm{i} \epsilon\right)^{2}}\left\{S_{\mu \nu}{ }^{\alpha \lambda} O_{\alpha}^{\mathrm{tw} 2}(\kappa x,-\kappa x)-\epsilon_{\mu \nu}^{\alpha \lambda} O_{5 \alpha}^{\mathrm{tw} 2}(\kappa x,-\kappa x)\right\},
\end{aligned}
$$

with

$$
\Omega_{(5) \alpha}^{\mathrm{tw} 2}(\kappa x,-\kappa x)=\partial_{\alpha} \int_{0}^{1} d \tau \int \frac{d^{4} u}{(2 \pi)^{4}} \Omega_{(5) \mu}(u)\left\{x^{\mu}(2+x \partial)-\frac{1}{2} \mathrm{i} \kappa \tau u^{\mu} x^{2}\right\}(3+x \partial) \mathcal{H}_{2}(u, \kappa \tau x),
$$

cf. Eqs. (2.222.23), and

$$
\mathcal{H}_{\nu}(u, \kappa x)=\sqrt{\pi}\left(\kappa \sqrt{(u x)^{2}-u^{2} x^{2}}\right)^{1 / 2-\nu} J_{\nu-1 / 2}\left(\frac{\kappa}{2} \sqrt{(u x)^{2}-u^{2} x^{2}}\right) e^{i \kappa(x u) / 2},
$$

and $\kappa=1 / 2$. In these relations a (formal) Fourier transform of the non-local operators is introduced and used off-cone later, i.e.,

$$
\left.\Omega_{(5) \mu}(\kappa \tilde{x},-\kappa \tilde{x})\right|_{\tilde{x} \rightarrow x}=\frac{1}{(2 \pi)^{4}} \int d^{4} u \Omega_{(5) \mu}(u) \mathrm{e}^{i \kappa(x u)} .
$$

Let us emphasize first that (2.27) is an off-cone operator equation which determines the twist2 part of $\Omega_{(5) \mu}(\kappa x,-\kappa x)$ by acting on $\mathrm{e}^{i \kappa(x u)}$ with a corresponding twist-2 projection operator $P_{\alpha}^{(2) \mu}\left(x, \partial_{x}\right)$ whose result is presented above. Second, it has been shown in [31] that, due to this projection and the structure of the Fourier kernel, from the operator $\Omega_{(5) \mu}(u)$ in (2.27) only its twist-2 part remains.

To calculate the twist-2 part of the Compton amplitude $T_{\mu \nu}(q)$ we have to parameterize the non-perturbative expectation values of the twist-2 operators $O_{(5) \mu}^{\mathrm{tw} 2}(\kappa x,-\kappa x)$. Here, following the general approach of [31], this is done by requiring

$$
\begin{aligned}
\left\langle p_{1},-p_{2}\right| e^{2} \mathrm{i}\left(\Omega_{\mu}(u)-\right. & \left.\Omega_{\mu}(-u)\right)\left|p_{1},-p_{2}\right\rangle \\
& =\sum_{a} \mathcal{K}_{\mu}^{a}\left(p_{ \pm}\right) \int D \mathbb{Z} \delta^{(4)}\left(u-p_{-} z_{-}-p_{+} z_{+}\right) f_{a}\left(z_{-}, z_{+}, t\right), \\
\left\langle p_{1},-p_{2}, S\right| e^{2}\left(\Omega_{5 \mu}(u)+\right. & \left.\Omega_{5 \mu}(-u)\right)\left|p_{1},-p_{2}, S\right\rangle \\
& =\sum_{a} \mathcal{K}_{5 \mu}^{a}\left(p_{ \pm}, S\right) \int D \mathbb{Z} \delta^{(4)}\left(u-p_{-} z_{-}-p_{+} z_{+}\right) f_{5 a}\left(z_{-}, z_{+}, t\right),
\end{aligned}
$$

where, in fact, under the $u$-integration the generalized distribution amplitudes $f_{(5) a}\left(z_{-}, z_{+} ; t\right)$ are reduced to their twist -2 contributions. These generalized distribution amplitudes depend 
explicitly on $t$, and additionally there is a $t$ - and $M^{2}$-dependence which finally results through the distribution $\delta^{(4)}\left(u-p_{-} z_{-}-p_{+} z_{+}\right)$from the Fourier transform in (2.26), together with (2.27. 2.30, 2.31), cf. Section 3 and 4 , as well as from the kinematic pre-factors $\mathcal{K}_{(5) \mu}^{a}\left(p_{ \pm}, S\right) .{ }^{2}$ Here, for the symmetric part of the hadronic tensor we choose $\mathcal{K}_{\mu}^{a}\left(p_{ \pm}\right)$as kinematic pre-factors

$$
\mathcal{K}^{1 \mu}=p_{-}^{\mu}, \quad \mathcal{K}^{2 \mu}=\pi_{-}^{\mu} \equiv p_{+}^{\mu}-\frac{p_{-}^{\mu}}{\eta}
$$

For the antisymmetric case the kinematic factors

$$
\mathcal{K}_{5}^{1 \mu}=S^{\mu}, \quad \mathcal{K}_{5}^{2 \mu}=p_{-}^{\mu} \frac{\left(p_{2} S\right)}{M^{2}}, \quad \mathcal{K}_{5}^{3 \mu}=\pi_{-}^{\mu} \frac{\left(p_{2} S\right)}{M^{2}}
$$

contribute. The normalization by $M^{2}$ in (2.33) is arbitrary and has to be arranged with the definition of the corresponding non-perturbative distribution functions. Furthermore, $f_{(5) a}\left(z_{-}, z_{+} ; t\right) \equiv f_{(5) a}(\mathbb{Z} ; t)$ denote the respective 2 -particle amplitudes characterized by the two fractions $z_{ \pm}$of momenta $p_{ \pm}$and a relative kinematic separation in $t$. The momentum fractions of the incoming $\left(z_{1}\right)$ and outgoing $\left(z_{2}\right)$ nucleon are formally unified into a 2-vector,

$$
\mathbb{Z}=\left(z_{+}, z_{-}\right)=\left(\left(z_{2}+z_{1}\right) / 2,\left(z_{2}-z_{1}\right) / 2\right)
$$

with the measure

$$
D \mathbb{Z}=2 d z_{+} d z_{-} \theta\left(1-z_{+}+z_{-}\right) \theta\left(1+z_{+}-z_{-}\right) \theta\left(1-z_{+}-z_{-}\right) \theta\left(1+z_{+}+z_{-}\right) .
$$

Due to the $\delta$-distribution in (2.30, 2.31) and the structure of the Compton amplitude, which is determined by (2.26 2.28), it appears convenient also to unify the momenta as

$$
\mathbb{P} \equiv\left(p_{+}, p_{-}\right)=\left(p_{2}+p_{1}, p_{2}-p_{1}\right)
$$

and to abbreviate expressions according to

$$
\Pi^{\mu}=\kappa \mathbb{P}^{\mu} \mathbb{Z}=\kappa\left(p_{+}^{\mu} z_{+}+p_{-}^{\mu} z_{-}\right), \quad \Pi_{\mu}^{\mathrm{T}}=g_{\mu \nu}^{\mathrm{T}} \Pi^{\nu}=\Pi_{\mu}-q_{\mu} \frac{(q \Pi)}{q^{2}}
$$

which replaces $\kappa u$ after performing the $u$-integration. Note that, due to the square roots in $\mathcal{H}_{2}(\kappa u, x)$, the Fourier transform can only be performed if $\Pi^{2}>0$ since $\sqrt{(q \Pi)^{2}-q^{2} \Pi^{2}}$ has to be real. In fact, this is equivalent to the requirement

$$
z_{+}^{2}+\frac{t z_{-}^{2}}{\left(4 M^{2}-t\right)} \geq 0,
$$

which restricts the allowed values of $z_{ \pm}$as a support condition to the generalized parton distributions $f_{(5) a}(\mathbb{Z}, t)$. It is convenient to introduce a common scale $\vartheta$ for the integration variables $z_{+}=\vartheta \zeta, z_{-}=\vartheta(1-\zeta / \eta)$, i.e., to change them non-linearly into $\vartheta$ and $\zeta$,

$$
\vartheta=z_{-}+\frac{z_{+}}{\eta}, \quad \zeta=\frac{z_{+}}{\vartheta} .
$$

Clearly, the $|t|$ values emerging in practice are not expected to deviate from this condition due to the presence of a non-perturbative damping factor $\exp (-b|t|)$ in the distribution functions, with

\footnotetext{
${ }^{2}$ In the following the explicit $t$-dependence of the distribution functions is understood also when we drop this variables in the respective expressions for brevity.
} 
$b \simeq 4 \ldots 8 \mathrm{GeV}^{-2}$. Connected to the support condition the positivity of the variable $\Pi^{2}(2.47)$ is guaranteed which is essential for performing Fourier transforms. The measure $D \mathbb{Z}$ in these new variables reads

$$
\begin{array}{r}
D \mathbb{Z}=2|\vartheta| d \vartheta d \zeta \theta(1-\vartheta+(1+1 / \eta) \vartheta \zeta) \theta(1+\vartheta-(1+1 / \eta) \vartheta \zeta) \\
\theta(1-\vartheta-(1-1 / \eta) \vartheta \zeta) \theta(1+\vartheta+(1-1 / \eta) \vartheta \zeta)
\end{array} .
$$

The parameterization of the matrix elements (2.30) and (2.31) is given by

$$
\begin{aligned}
\left\langle p_{1},-p_{2}\left|e^{2} \mathrm{i}\left(\Omega_{\mu}(u)-\Omega_{\mu}(u)\right)\right| p_{1},-p_{2}\right\rangle & =\sum_{a} \mathcal{K}_{\mu}^{a}\left(p_{ \pm}\right) \int D \mathbb{Z} \delta(u-\vartheta \mathcal{P}) f_{a}(\vartheta, \zeta ; t),(2.41) \\
\left\langle p_{1},-p_{2}, S\left|e^{2}\left(\Omega_{5 \mu}(u)+\Omega_{5 \mu}(u)\right)\right| p_{1},-p_{2}, S\right\rangle & =\sum_{a} \mathcal{K}_{5 \mu}^{a}\left(p_{ \pm}, S\right) \int D \mathbb{Z} \delta(u-\vartheta \mathcal{P}) f_{5 a}(\vartheta, \zeta ; t) .
\end{aligned}
$$

In the following we make the $\vartheta$-dependence of the kinematics explicit :

$$
\begin{aligned}
\Pi^{\mu} & =\kappa \vartheta \mathcal{P}^{\mu}(\eta ; \zeta), \\
\mathcal{P}^{\mu}(\eta, \zeta) & =p_{-}^{\mu}+\pi_{-}^{\mu} \zeta=p_{-}^{\mu}(1-\zeta / \eta)+p_{+}^{\mu} \zeta, \\
q \Pi & =\kappa \vartheta(q \mathcal{P}), \\
(q \mathcal{P}) & =\left(q p_{-}\right)=q^{2} / 2 \beta \leq 0, \\
\Pi^{2} & =(\kappa \vartheta)^{2} \mathcal{P}^{2}, \\
\mathcal{P}^{2} & =t(1-\zeta / \eta)^{2}+\left(4 M^{2}-t\right) \zeta^{2} \geq 0, \\
\left(\Pi^{\mathrm{T}}\right)^{2} & =(\kappa \vartheta)^{2}\left(\mathcal{P}^{\mathrm{T}}\right)^{2}=(\kappa \vartheta)^{2} \frac{(q \mathcal{P})^{2}-q^{2} \mathcal{P}^{2}}{-q^{2}} \\
\frac{\mathcal{P}^{2}}{\left(\mathcal{P}^{\mathrm{T}}\right)^{2}} & =\frac{4 \beta^{2} \mathcal{P}^{2} / Q^{2}}{1+4 \beta^{2} \mathcal{P}^{2} / Q^{2}} \geq 0 .
\end{aligned}
$$

$\mathcal{P}^{2}$ depends on both $t$ and the integration variable $\zeta$ which is limited by the Heaviside functions included in the measure (2.40).

Recall, that $\mathcal{P}^{2} \geq 0$ is required which for $t=0$ is trivially fulfilled. Concerning the region of small values of $t<0$ one observes that $\zeta=0$ is to be excluded.

As will be shown in Sections 3 and 4, the absorptive part of the Compton amplitude solely emerges from a factor $1 /[R(1)+\mathrm{i} \epsilon]$ with the polynomial

$$
R(\tau)=(q+\tau \Pi)^{2}=\left(\tau^{2} \Pi^{2}+2 \tau q \Pi+q^{2}\right)=\Pi^{2}\left(\tau-\tilde{\xi}_{+}\right)\left(\tau-\tilde{\xi}_{-}\right) .
$$

The roots are

$$
\tilde{\xi}_{ \pm}=\frac{-q \Pi \pm \sqrt{(q \Pi)^{2}-q^{2} \Pi^{2}}}{\Pi^{2}}=-\frac{q^{2}}{q \Pi \pm \sqrt{(q \Pi)^{2}-q^{2} \Pi^{2}}}
$$

leading to

$$
\frac{1}{R(\tau)+\mathrm{i} \epsilon}=\frac{1}{2 \sqrt{(q \Pi)^{2}-q^{2} \Pi^{2}}}\left(\frac{1}{\tau-\tilde{\xi}_{+}+\mathrm{i} \epsilon}-\frac{1}{\tau-\tilde{\xi}_{-}-\mathrm{i} \epsilon}\right) .
$$

The imaginary part of the Compton amplitude results thus from

$$
\operatorname{Im} \frac{1}{R(1)+\mathrm{i} \epsilon}=-\frac{\pi}{2} \frac{1}{\sqrt{(q \Pi)^{2}-q^{2} \Pi^{2}}}\left[\delta\left(1-\tilde{\xi}_{+}\right)+\delta\left(1-\tilde{\xi}_{-}\right)\right] .
$$


Only the term containing the variable $\tilde{\xi}_{+}$contributes to the cross section, whereas there is no contribution due to the term $\tilde{\xi}_{-}$. This is seen as follows. For negative values of $\vartheta, 1 / \eta \leq \vartheta \leq 0$, $(q \Pi) \geq 0$, holds and therefore, in terms of variables $(\vartheta, \zeta)$, one obtains

$$
\begin{aligned}
& \tilde{\xi}_{+}=-\frac{2 \beta}{\kappa \vartheta} \frac{1}{1+\sqrt{1+4 \beta^{2} \mathcal{P}^{2} / Q^{2}}} \Longrightarrow-\frac{\beta}{\kappa \vartheta} \geq 0 \\
& \tilde{\xi}_{-}=-\frac{2 \beta}{\kappa \vartheta} \frac{1}{1-\sqrt{1+4 \beta^{2} \mathcal{P}^{2} / Q^{2}}} \Longrightarrow \frac{1}{\kappa \beta \vartheta} \frac{Q^{2}}{\mathcal{P}^{2}} \rightarrow-\infty
\end{aligned}
$$

taking into account the support condition for $\mathcal{P}(\zeta)$. Here $\Longrightarrow$ denotes the limit $Q^{2} \rightarrow \infty$. Therefore $\delta\left(1-\tilde{\xi}_{-}\right)$does not contribute. In the case of positive $\vartheta, 0 \leq \vartheta \leq-1 / \eta$, one finds

$$
\begin{aligned}
& \tilde{\xi}_{+}=-\frac{2 \beta}{\kappa \vartheta} \frac{1}{1+\sqrt{1+4 \beta^{2} \mathcal{P}^{2} / Q^{2}}} \Longrightarrow \frac{1}{\kappa \beta \vartheta} \frac{Q^{2}}{\mathcal{P}^{2}} \rightarrow+\infty \\
& \tilde{\xi}_{-}=-\frac{2 \beta}{\kappa \vartheta} \frac{1}{1-\sqrt{1+4 \beta^{2} \mathcal{P}^{2} / Q^{2}}} \Longrightarrow-\frac{\beta}{\kappa \vartheta} \leq 0,
\end{aligned}
$$

and neither $\delta\left(1-\tilde{\xi}_{+}\right)$nor $\delta\left(1-\tilde{\xi}_{-}\right)$contribute.

Having this in mind, let us rewrite

$$
\tilde{\xi}_{+}=\frac{\xi}{\vartheta}, \quad \xi=-\frac{2 \beta}{\kappa} \frac{1}{1+\sqrt{1+4 \beta^{2} \mathcal{P}^{2} / Q^{2}}}=\frac{1}{2 \kappa \beta} \frac{Q^{2} / \mathcal{P}^{2}}{1-\sqrt{1+4 \beta^{2} \mathcal{P}^{2} / Q^{2}}}
$$

from which it is evident that in case of diffractive scattering $-2 \beta$ takes the role of Bjorken variable $x_{B j}$ in deep inelastic scattering as $\mathcal{P}^{2}$ takes the role of $4 M^{2}$. Furthermore, rewriting

$$
\delta\left(1-\tilde{\xi}_{+}\right)=|\vartheta| \delta(\vartheta-\xi)
$$

it is obvious that after integration over $\vartheta$ that variable is replaced by $\xi$ everywhere. For intermediate momenta $\xi$ substitutes the scaling variable $\beta$ and plays the role of a generalized Nachtmann variable. Let us note that this variable, however, implicitly depends on $\zeta, \beta$ and $\eta$ through $\mathcal{P}(\zeta)$.

Due to Eqs. (2.52), the following equalities

$$
\left(\Pi^{2}+q \Pi\right) \delta\left(1-\tilde{\xi}_{+}\right)=\sqrt{(q \Pi)^{2}-q^{2} \Pi^{2}} \delta\left(1-\tilde{\xi}_{+}\right)=-\left(q^{2}+q \Pi\right) \delta\left(1-\tilde{\xi}_{+}\right)
$$

hold and can be used to simplify some expressions resulting for the absorptive part of the Compton amplitude.

\section{The Symmetric Part of the Amplitude}

In the case of unpolarized scattering the symmetric part of the Fourier transform of the operator $x_{\alpha} O_{\beta}^{\mathrm{tw} 2}(\kappa x,-\kappa x) /\left(x^{2}-\mathrm{i} \epsilon\right)^{2}$ determines the contribution to the hadronic tensor of diffractive scattering. It reads (cf. [27], Eq. (6.1)),

$$
\begin{aligned}
\widehat{T}_{\{\mu \nu\}}^{\mathrm{tw} 2}(q)= & S_{\mu \nu \mid}^{\alpha \beta} \int \frac{d^{4} x}{2 \pi^{2}} \frac{\mathrm{e}^{\mathrm{i} q x}}{\left(x^{2}-\mathrm{i} \epsilon\right)^{2}} x_{\alpha} i e^{2}\left(\Omega_{\beta}^{\mathrm{tw} 2}(\kappa x,-\kappa x)-\Omega_{\beta}^{\mathrm{tw} 2}(-\kappa x, \kappa x)\right) \\
= & 2 i e^{2} \int_{0}^{1} d \tau(1-\tau+\tau \ln \tau) \int \frac{d^{4} u}{\kappa^{4} \tau^{4}}\left(\Omega^{\rho}\left(\frac{u}{\kappa \tau}\right)-\Omega^{\rho}\left(\frac{-u}{\kappa \tau}\right)\right) \partial_{\rho}^{u} \times \\
& {\left[\frac{2\left(q^{2}\right)^{2}}{\left[(q+u)^{2}+i \epsilon\right]^{3}}\left(u_{\mu}^{\mathrm{T}} u_{\nu}^{\mathrm{T}}-g_{\mu \nu}^{\mathrm{T}}\left(u^{\mathrm{T}}\right)^{2}\right)+\frac{u^{2} q^{2}}{\left[(q+u)^{2}+i \epsilon\right]^{2}} g_{\mu \nu}^{\mathrm{T}}\right] . }
\end{aligned}
$$


We have to form matrix elements $\left\langle p_{1}-p_{2}\left|\widehat{T}_{\{\mu \nu\}}^{\mathrm{tw} 2}(q)\right| p_{1},-p_{2}\right\rangle$ for this expression using the nonperturbative matrix elements (2.41) of the operator $i(\Omega(u)-\Omega(-u))$. This representation of the operator matrix element, based on the generalized parton distribution functions $f_{a}(\mathbb{Z})$, has to be inserted into the Fourier transform of the Compton operator.

After a straightforward, but tedious calculation one obtains for the part which contains the factor $1 /(R(1)+\mathrm{i} \varepsilon)$, see [27], Eq. (6.4),

$$
\begin{aligned}
T_{\{\mu \nu\}}^{\mathrm{tw} 2}(q)= & \frac{q^{2}}{2} \int D \mathbb{Z}\left\{\frac{q \mathcal{K}_{a}}{q \Pi}\left[g_{\mu \nu}^{\mathrm{T}} F_{1}^{a}(\mathbb{Z})-\frac{\Pi_{\mu}^{\mathrm{T}} \Pi_{\nu}^{\mathrm{T}}}{\left(\Pi^{\mathrm{T}}\right)^{2}} F_{2}^{a}(\mathbb{Z})\right]\right. \\
& +\left(\frac{q \mathcal{K}_{a}}{q \Pi}-\frac{\Pi_{\mathcal{K}}}{\Pi^{2}}\right)\left[g_{\mu \nu}^{\mathrm{T}} F_{3}^{a}(\mathbb{Z})-\frac{\Pi_{\mu}^{\mathrm{T}} \Pi_{\nu}^{\mathrm{T}}}{\left(\Pi^{\mathrm{T}}\right)^{2}} F_{4}^{a}(\mathbb{Z})\right] \\
& \left.-\left(\frac{\mathcal{K}_{a \mu}^{\mathrm{T}} \Pi_{\nu}^{\mathrm{T}}+\Pi_{\mu}^{\mathrm{T}} \mathcal{K}_{a \nu}^{\mathrm{T}}}{\left(\Pi^{\mathrm{T}}\right)^{2}}-2 \frac{q \mathcal{K}_{a}}{q \Pi} \frac{\Pi_{\mu}^{\mathrm{T}} \Pi_{\nu}^{\mathrm{T}}}{\left(\Pi^{\mathrm{T}}\right)^{2}}\right) F_{5}^{a}(\mathbb{Z})\right\} \frac{1}{R(1)+\mathrm{i} \epsilon} .
\end{aligned}
$$

In principle there occur also terms $\propto 1 /(R(0)+\mathrm{i} \varepsilon)$ which, due to the overall factor $q^{2}$, do not contribute to the imaginary part and are therefore omitted here. Obviously, the whole expression obeys transversality proving gauge invariance of the complete amplitude. The functions $F^{a}(\mathbb{Z} ; q, \Pi)$ are

$$
\begin{aligned}
F_{1}^{a}(\mathbb{Z})= & f^{a}(\mathbb{Z})+\frac{\Pi^{2}\left(q \Pi+\Pi^{2}\right)}{(q \Pi)^{2}-q^{2} \Pi^{2}} \int_{0}^{1} \frac{d \tau}{\tau^{2}} f^{a}\left(\frac{\mathbb{Z}}{\tau}\right)+\frac{\left[\Pi^{2}\right]^{2}}{(q \Pi)^{2}-q^{2} \Pi^{2}} \int_{0}^{1} \frac{d \tau}{\tau^{2}} \int_{0}^{1} \frac{d \tau_{1}}{\tau_{1}^{3}} f^{a}\left(\frac{\mathbb{Z}}{\tau \tau_{1}}\right), \\
F_{2}^{a}(\mathbb{Z})= & f^{a}(\mathbb{Z})+\frac{3 \Pi^{2}\left(q \Pi+\Pi^{2}\right)}{(q \Pi)^{2}-q^{2} \Pi^{2}} \int_{0}^{1} \frac{d \tau}{\tau^{2}} f^{a}\left(\frac{\mathbb{Z}}{\tau}\right)+\frac{3\left[\Pi^{2}\right]^{2}}{(q \Pi)^{2}-q^{2} \Pi^{2}} \int_{0}^{1} \frac{d \tau}{\tau^{2}} \int_{0}^{1} \frac{d \tau_{1}}{\tau_{1}^{3}} f^{a}\left(\frac{\mathbb{Z}}{\tau \tau_{1}}\right) \\
= & 3 F_{1}^{a}-2 f^{a}(\mathbb{Z}), \\
F_{3}^{a}(\mathbb{Z})= & -\int_{0}^{1} \frac{d \tau}{\tau^{2}}\left[F_{1}^{a}\left(\frac{\mathbb{Z}}{\tau}\right)+\frac{(q \Pi)^{2}}{(q \Pi)^{2}-q^{2} \Pi^{2}} F_{2}^{a}\left(\frac{\mathbb{Z}}{\tau}\right)\right]-\frac{\Pi^{2}(q+\Pi)^{2}}{(q \Pi)^{2}-q^{2} \Pi^{2}} \int_{0}^{1} \frac{d \tau}{\tau^{2}} f^{a}\left(\frac{\mathbb{Z}}{\tau}\right) \\
& +\frac{2(q \Pi)}{(q \Pi)^{2}-q^{2} \Pi^{2}} \int_{0}^{1} \frac{d \tau}{\tau^{2}}\left[\left(q \Pi+\Pi^{2}\right) f^{a}\left(\frac{\mathbb{Z}}{\tau}\right)+\Pi^{2} \int_{0}^{1} \frac{d \tau_{1}}{\tau_{1}^{2}} f^{a}\left(\frac{\mathbb{Z}}{\tau \tau_{1}}\right)\right] \\
& +\frac{\Pi^{2}}{(q \Pi)^{2}-q^{2} \Pi^{2}}\left[\left(q \Pi+q^{2}\right) f^{a}(\mathbb{Z})+(q \Pi) \int_{0}^{1} \frac{d \tau}{\tau^{2}} f^{a}\left(\frac{\mathbb{Z}}{\tau}\right)\right], \\
F_{4}^{a}(\mathbb{Z})= & 3 F_{3}^{a}+2 \frac{\Pi^{2}}{\left(\Pi^{\mathrm{T}}\right)^{2}} F_{5}^{a}-2 \frac{\Pi^{2}}{(q \Pi)^{2}-q^{2} \Pi^{2}}\left[\left(q \Pi+q^{2}\right) f^{a}(\mathbb{Z})+(q \Pi) \int_{0}^{1} \frac{d \tau}{\tau^{2}} f^{a}\left(\frac{\mathbb{Z}}{\tau}\right)\right],(3.6) \\
F_{5}^{a}(\mathbb{Z})= & \int_{0}^{1} \frac{d \tau}{\tau^{2}} F_{2}^{a}\left(\frac{\mathbb{Z}}{\tau}\right) .
\end{aligned}
$$

The imaginary part of the Compton amplitude is given by

$$
\begin{aligned}
\operatorname{Im} T_{\{\mu \nu\}}^{\mathrm{tw} 2}(q)= & \frac{-\pi}{2 \kappa^{2}} \int d \zeta \int_{1 / \eta}^{0} d \vartheta \frac{q^{2}}{\left[(q \mathcal{P})^{2}-q^{2} \mathcal{P}^{2}\right]^{1 / 2}} \delta(\vartheta-\xi) \\
& \times\left\{\frac{q \mathcal{K}_{a}}{q \mathcal{P}}\left[g_{\mu \nu}^{\mathrm{T}} F_{1}^{a}(\vartheta, \zeta)-\frac{\mathcal{P}_{\mu}^{\mathrm{T}} \mathcal{P}_{\nu}^{\mathrm{T}}}{\left(\mathcal{P}^{\mathrm{T}}\right)^{2}} F_{2}^{a}(\vartheta, \zeta)\right]\right. \\
& +\left(\frac{q \mathcal{K}_{a}}{q \mathcal{P}}-\frac{\mathcal{P} \mathcal{K}_{a}}{\mathcal{P}^{2}}\right)\left[g_{\mu \nu}^{\mathrm{T}} F_{3}^{a}(\vartheta, \zeta)-\frac{\mathcal{P}_{\mu}^{\mathrm{T}} \mathcal{P}_{\nu}^{\mathrm{T}}}{\left(\mathcal{P}^{\mathrm{T}}\right)^{2}} F_{4}^{a}(\vartheta, \zeta)\right] \\
& \left.-\left(\frac{\mathcal{K}_{a \mu}^{\mathrm{T}} \mathcal{P}_{\nu}^{\mathrm{T}}+\mathcal{P}_{\mu}^{\mathrm{T}} \mathcal{K}_{a \nu}^{\mathrm{T}}}{\left(\mathcal{P}^{\mathrm{T}}\right)^{2}}-2 \frac{q \mathcal{K}_{a}}{q \mathcal{P}} \frac{\mathcal{P}_{\mu}^{\mathrm{T}} \mathcal{P}_{\nu}^{\mathrm{T}}}{\left(\mathcal{P}^{\mathrm{T}}\right)^{2}}\right) F_{5}^{a}(\vartheta, \zeta)\right\} .
\end{aligned}
$$


We change now to the variables $(\vartheta, \zeta)$ and express the above integrals using the following new distribution amplitudes :

$$
\begin{aligned}
\Phi_{a}^{(0)}(\vartheta, \zeta) & \equiv f_{a}(\vartheta, \zeta) \\
\Phi_{a}^{(1)}(\vartheta, \zeta) & \equiv \int_{\vartheta}^{1 / \eta} d y_{1} f_{a}\left(y_{1}, \zeta\right)=\vartheta \int_{0}^{1} \frac{d \tau}{\tau^{2}} f_{a}\left(\frac{\vartheta}{\tau}, \zeta\right), \\
\Phi_{a}^{(2)}(\vartheta, \zeta) & \equiv \int_{\vartheta}^{1 / \eta} d y_{2} \int_{y_{2}}^{1 / \eta} d y_{1} f_{a}\left(y_{1}, \zeta\right)=\vartheta^{2} \int_{0}^{1} \frac{d \tau_{1}}{\tau_{1}^{3}} \int_{0}^{1} \frac{d \tau_{2}}{\tau_{2}^{2}} f_{a}\left(\frac{\vartheta}{\tau_{1} \tau_{2}}, \zeta\right), \\
\Phi_{a}^{(i)}(\vartheta, \zeta) & \equiv \int_{\vartheta}^{1 / \eta} d y \Phi_{a}^{(i-1)}(y, \zeta) .
\end{aligned}
$$

In the following we consider the imaginary part of the Compton amplitude which forms the hadronic tensor for deep-inelastic diffractive scattering. As already stated above, the imaginary part follows from $\operatorname{Im}\{1 /(R+i \epsilon)\}$. The $\vartheta$ integral is easily carried out

$$
\begin{aligned}
\operatorname{Im} T_{\{\mu \nu\}}^{\mathrm{tw} 2}(q)= & \frac{-\pi}{2 \kappa^{2}} \int d \zeta \frac{q^{2}}{\left[(q \mathcal{P})^{2}-q^{2} \mathcal{P}^{2}\right]^{1 / 2}} \\
& \times\left\{\frac{q \mathcal{K} \mathcal{K}_{a}}{q \mathcal{P}}\left[g_{\mu \nu}^{\mathrm{T}} F_{1}^{a}(\xi, \zeta)-\frac{\mathcal{P}_{\mu}^{\mathrm{T}} \mathcal{P}_{\nu}^{\mathrm{T}}}{\left(\mathcal{P}^{\mathrm{T}}\right)^{2}} F_{2}^{a}(\xi, \zeta)\right]\right. \\
& +\left(\frac{q \mathcal{K}_{a}}{q \mathcal{P}}-\frac{\mathcal{P} \mathcal{K}_{a}}{\mathcal{P}^{2}}\right)\left[g_{\mu \nu}^{\mathrm{T}} F_{3}^{a}(\xi, \zeta)-\frac{\mathcal{P}_{\mu}^{\mathrm{T}} \mathcal{P}_{\nu}^{\mathrm{T}}}{\left(\mathcal{P}^{\mathrm{T}}\right)^{2}} F_{4}^{a}(\xi, \zeta)\right] \\
& \left.-\left(\frac{\mathcal{K}_{a \mu}^{\mathrm{T}} \mathcal{P}_{\nu}^{\mathrm{T}}+\mathcal{P}_{\mu}^{\mathrm{T}} \mathcal{K}_{a \nu}^{\mathrm{T}}}{\left(\mathcal{P}^{\mathrm{T}}\right)^{2}}-2 \frac{q \mathcal{K}_{a}}{q \mathcal{P}} \frac{\mathcal{P}_{\mu}^{\mathrm{T}} \mathcal{P}_{\nu}^{\mathrm{T}}}{\left(\mathcal{P}^{\mathrm{T}}\right)^{2}}\right) F_{5}^{a}(\xi, \zeta)\right\},
\end{aligned}
$$

where the substitution $\Pi_{\mu}=\kappa \xi \mathcal{P}_{\mu}$ was used. Here the functions $F_{i}^{a}(\mathbb{Z} ; q, \Pi) \equiv$ $F_{i}^{a}(\xi, \zeta ; q, \kappa \xi \mathcal{P}), i=1, \ldots, 5$, are obtained from (3.3) - (3.7) using (2.61) in a non-trivial way :

$$
\begin{aligned}
F_{1}^{a}(\xi, \zeta) \equiv & \Phi_{a}(\xi, \zeta)+\frac{\kappa \mathcal{P}^{2}}{\left[(q \mathcal{P})^{2}-q^{2} \mathcal{P}^{2}\right]^{1 / 2}} \Phi_{a}^{1}(\xi, \zeta)+\frac{\kappa^{2}\left[\mathcal{P}^{2}\right]^{2}}{(q \mathcal{P})^{2}-q^{2} \mathcal{P}^{2}} \Phi_{a}^{2}(\xi, \zeta) \\
F_{2}^{a}(\xi, \zeta) \equiv & \Phi_{a}(\xi, \zeta)+\frac{3 \kappa \mathcal{P}^{2}}{\left[(q \mathcal{P})^{2}-q^{2} \mathcal{P}^{2}\right]^{1 / 2}} \Phi_{a}^{1}(\xi, \zeta)+\frac{\left.3 \kappa^{2} \mathcal{P}^{2}\right]^{2}}{(q \mathcal{P})^{2}-q^{2} \mathcal{P}^{2}} \Phi_{a}^{2}(\xi, \zeta) \\
F_{a 3}(\xi, \zeta) \equiv & -\int_{0}^{1} \frac{d \tau}{\tau^{2}}\left[F_{a 1}\left(\frac{\xi}{\tau}, \zeta\right)+\frac{(q \mathcal{P})^{2}}{(q \mathcal{P})^{2}-q^{2} \mathcal{P}^{2}} F_{a 2}\left(\frac{\xi}{\tau}, \zeta\right)\right] \\
& +\frac{2(q \mathcal{P})}{\left[(q \mathcal{P})^{2}-q^{2} \mathcal{P}^{2}\right]^{1 / 2}} \int_{0}^{1} \frac{d \tau}{\tau^{2}}\left[\Phi_{a}^{(0)}\left(\frac{\xi}{\tau}, \zeta\right)+\frac{\kappa \mathcal{P}^{2}}{\left[(q \mathcal{P})^{2}-q^{2} \mathcal{P}^{2}\right]^{1 / 2}} \Phi_{a}^{(1)}\left(\frac{\xi}{\tau}, \zeta\right)\right] \\
& +\frac{\kappa \mathcal{P}^{2}}{\left[(q \mathcal{P})^{2}-q^{2} \mathcal{P}^{2}\right]^{1 / 2}}\left[-\xi \Phi_{a}^{(0)}(\xi, \zeta)+\frac{q \mathcal{P}}{\left[(q \mathcal{P})^{2}-q^{2} \mathcal{P}^{2}\right]^{1 / 2}} \Phi_{a}^{(1)}(\xi, \zeta)\right] \\
F_{a 4}(\xi, \zeta) \equiv & 3 F_{a 3}(\xi, \zeta)-\frac{2 q^{2} \mathcal{P}^{2}}{\left[(q \mathcal{P})^{2}-q^{2} \mathcal{P}^{2}\right]} F_{a 5}(\xi, \zeta) \\
& -\frac{2 \kappa \mathcal{P}^{2}}{\left[(q \mathcal{P})^{2}-q^{2} \mathcal{P}^{2}\right]^{1 / 2}}\left[-\xi \Phi_{a}^{(0)}(\xi, \zeta)+\frac{q \mathcal{P}}{\left[(q \mathcal{P})^{2}-q^{2} \mathcal{P}^{2}\right]^{1 / 2}} \Phi_{a}^{(1)}(\xi, \zeta)\right] \\
F_{a 5}(\xi, \zeta) \equiv & \int_{0}^{1} \frac{d \tau}{\tau^{2}} F_{a 2}\left(\frac{\xi}{\tau}, \zeta\right) \cdot
\end{aligned}
$$


In the limit $t, M^{2} \rightarrow 0$, Eqs. (5,8) of Ref. [7], the hadronic tensor simplifies and contains only two structure functions which are linear combinations of the four occurring in the general case (2.10) weighted by powers of $\left(1-x_{\mathbb{P}}\right)$. In this limit the functions $\left.F_{k}^{a}\right|_{k=3} ^{5}$ do not vanish and contribute to the two structure functions remaining. On the other hand, $\left.F_{k}^{a}\right|_{k=3} ^{5}$ do not contribute for forward scattering $p_{2} \rightarrow 0$.

Now, we can rewrite the imaginary part of the Compton amplitude, simply having recourse to [27], Eqs. (6.27) and (6.36), and replacing there $x \rightarrow-2 \beta$ :

$$
\operatorname{Im} T_{\{\mu \nu\}}^{\mathrm{tw} 2}(q)=\operatorname{Im} T_{1\{\mu \nu\}}^{\mathrm{tw} 2}(q)+\operatorname{Im} T_{2\{\mu \nu\}}^{\mathrm{tw} 2}(q)
$$

where

$$
\begin{aligned}
\operatorname{Im} T_{1\{\mu \nu\}}^{\mathrm{tw} 2}(q)= & \sum_{a} 2 \pi \int d \zeta \frac{q \mathcal{K}_{a}}{q \mathcal{P}}\left[-g_{\mu \nu}^{\mathrm{T}} W_{a 1}^{\mathrm{diff}}(\xi, \beta, \eta ; \zeta)+\frac{\mathcal{P}_{\mu}^{\mathrm{T}} \mathcal{P}_{\nu}^{\mathrm{T}}}{\mathcal{P}^{2}} W_{a 2}^{\mathrm{diff}}(\xi, \beta, \eta ; \zeta)\right] \\
\operatorname{Im} T_{2\{\mu \nu\}}^{\mathrm{tw} 2}(q)= & \sum_{a} 2 \pi \int d \zeta\left\{\left(\frac{q \mathcal{K}_{a}}{q \mathcal{P}}-\frac{\mathcal{P} \mathcal{K}_{a}}{\mathcal{P}^{2}}\right)\left[g_{\mu \nu}^{\mathrm{T}}-3 \frac{\mathcal{P}_{\mu}^{\mathrm{T}} \mathcal{P}_{\nu}^{\mathrm{T}}}{\left(\mathcal{P}^{\mathrm{T}}\right)^{2}}\right]\right. \\
& \times\left[\int_{\xi}^{1 / \eta} \frac{d y}{\xi}\left(W_{a 1}^{\mathrm{diff}}(y, \beta, \eta ; \zeta)+\frac{Q^{2}}{4 \beta^{2} \mathcal{P}^{2}} W_{a 2}^{\mathrm{diff}}(y, \beta, \eta ; \zeta)\right)\right. \\
& \left.+\int_{\xi}^{1 / \eta} \frac{d y}{\xi}\left(2 V_{a 0}^{\mathrm{diff}}(y, \beta, \eta ; \zeta)+V_{a 1}^{\mathrm{diff}}(y, \beta, \eta ; \zeta)\right)\right] \\
& +\left(\mathcal{P}_{\mu}^{\mathrm{T}} g_{\nu \alpha}^{\mathrm{T}}+\mathcal{P}_{\nu}^{\mathrm{T}} g_{\mu \alpha}^{\mathrm{T}}-2 \frac{\mathcal{P}_{\mu}^{\mathrm{T}} \mathcal{P}_{\nu}^{\mathrm{T}}}{\left(\mathcal{P}^{\mathrm{T}}\right)^{2}} \mathcal{P}_{\alpha}^{\mathrm{T}}\right) \frac{\mathcal{K}_{a}^{\alpha}}{\mathcal{P}^{2}} \int_{\xi}^{1 / \eta} \frac{d y}{\xi} W_{a 2}^{\mathrm{diff}}(y, \beta, \eta ; \zeta) \\
& \left.+\frac{1}{2}\left(\frac{q \mathcal{\mathcal { K } _ { a }}}{q \mathcal{P}}-\frac{\mathcal{P \mathcal { K }}_{a}}{\mathcal{P}^{2}}\right)\left[g_{\mu \nu}^{\mathrm{T}}-\frac{\mathcal{P}_{\mu}^{\mathrm{T}} \mathcal{P}_{\nu}^{\mathrm{T}}}{\left(\mathcal{P}^{\mathrm{T}}\right)^{2}}\right] V_{a 1}^{\mathrm{diff}}(\xi, \beta, \eta ; \zeta)\right\}
\end{aligned}
$$

where

$$
\begin{aligned}
& W_{a 1}^{\text {diff }}(\xi, \beta, \eta ; \zeta) \equiv \frac{2 \beta}{\sqrt{1+4 \beta^{2} \mathcal{P}^{2} / Q^{2}}} F_{1}^{a}(\xi, \beta, \eta ; \zeta), \\
& W_{a 2}^{\text {diff }}(\xi, \beta, \eta ; \zeta) \equiv \frac{(2 \beta)^{3}}{\left(\sqrt{1+4 \beta^{2} \mathcal{P}^{2} / Q^{2}}\right)^{3}} F_{2}^{a}(\xi, \beta, \eta ; \zeta)
\end{aligned}
$$

and

$$
\begin{aligned}
V_{a 0}^{\text {diff }}(\xi, \beta, \eta ; \zeta) & \equiv \frac{-2 \beta \Phi_{a}^{(0)}(\xi ; \zeta)}{\sqrt{1+4 \beta^{2} \mathcal{P}^{2} / Q^{2}}}, \\
V_{a 1}^{\text {diff }}(\xi, \beta, \eta ; \zeta) & \equiv \frac{-2 \beta^{2} \mathcal{P}^{2}}{Q^{2}} \frac{\partial}{\partial \beta}\left(\frac{-2 \beta \Phi_{a}^{(1)}(\xi ; \zeta)}{\sqrt{1+4 \beta^{2} \mathcal{P}^{2} / Q^{2}}}\right) \\
& =\frac{4 \beta^{2} \mathcal{P}^{2} / Q^{2}}{1+4 \beta^{2} \mathcal{P}^{2} / Q^{2}}\left[-\xi \Phi_{a}^{(0)}(\xi, \zeta)+\frac{\Phi_{a}^{(1)}(\xi, \zeta)}{\sqrt{1+4 \beta^{2} \mathcal{P}^{2} / Q^{2}}}\right] .
\end{aligned}
$$

Note that $\xi=\xi(\beta, \eta ; \zeta)$ and $\mathcal{P}=\mathcal{P}(\eta ; \zeta)$. Differential operators as emerging in (3.24) are characteristic for target mass corrections and were applied earlier in Refs. [11,12], see also [23]. If the corresponding differential expressions are found the associated integral representations are derived straightforwardly. 
We now convert the result referring to bases like used in (2.10). By observing

$$
\frac{\mathcal{P}^{\mathrm{T}} \mathcal{K}_{a}^{\mathrm{T}}}{\mathcal{P}^{2}}=\frac{\mathcal{P} \mathcal{K}_{a}}{\mathcal{P}^{2}}+\frac{1}{4 \beta^{2} \mathcal{P}^{2} / Q^{2}} \frac{q \mathcal{K}_{a}}{q \mathcal{P}}
$$

one obtains

$$
\begin{aligned}
\frac{1}{2 \pi} \operatorname{Im} T_{\{\mu \nu\}}(q)= & \sum_{a} \int d \zeta\left[-g_{\mu \nu}^{\mathrm{T}}\left(\frac{q \mathcal{K}_{a}}{q \mathcal{P}} T_{1}^{a}(\xi, \zeta)+\frac{\mathcal{P} \mathcal{K}_{a}}{\mathcal{P}^{2}} T_{2}^{a}(\xi, \zeta)\right)\right. \\
& \left.+\frac{\mathcal{P}_{\mu}^{\mathrm{T}} \mathcal{P}_{\nu}^{\mathrm{T}}}{\left(\mathcal{P}^{\mathrm{T}}\right)^{2}}\left(\frac{q \mathcal{K}_{a}}{q \mathcal{P}} T_{3}^{a}(\xi, \zeta)+\frac{\mathcal{P} \mathcal{K}_{a}}{\mathcal{P}^{2}} T_{4}^{a}(\xi, \zeta)\right)+\frac{\mathcal{K}_{a \nu}^{\mathrm{T}} \mathcal{P}_{\mu}^{\mathrm{T}}+\mathcal{K}_{a \mu}^{\mathrm{T}} \mathcal{P}_{\nu}^{\mathrm{T}}}{\mathcal{P}^{2}} T_{5}^{a}(\xi, \zeta)\right] .
\end{aligned}
$$

The distribution functions $T_{i}^{a}(\xi, \zeta)$ are related to the distributions used above by

$$
\begin{aligned}
T_{1}^{a}(\xi, \zeta) & =W_{a 1}^{\text {diff }}(\xi, \beta, \eta ; \zeta)-T_{2}^{a}(\xi, \zeta), \\
T_{2}^{a}(\xi, \zeta) & =\int_{\xi}^{1 / \eta} \frac{d y}{\xi}\left[2 W_{a L}^{\text {diff }}-W_{a 2}^{\text {diff }}+V_{a 1}^{\text {diff }}\right](y, \beta, \eta ; \zeta)+\frac{1}{2} V_{a 1}^{\text {diff }}(\xi, \beta, \eta ; \zeta), \\
T_{3}^{a}(\xi, \zeta) & =\left(W_{a 1}^{\text {diff }}+W_{a L}^{\text {diff }}\right)(\xi, \beta, \eta ; \zeta)-\frac{Q^{2}}{2 \beta^{2} \mathcal{P}^{2}} \int_{\xi}^{1 / \eta} \frac{d y}{\xi} W_{a 2}^{\text {diff }}(y, \beta, \eta ; \zeta) \\
& -\left(3 T_{2}^{a}(\xi, \zeta)-V_{a 1}^{\text {diff }}(\xi, \beta, \eta ; \zeta)\right) \\
T_{4}^{a}(\xi, \zeta) & =-2 \int_{\xi}^{1 / \eta} \frac{d y}{\xi} W_{a 2}^{\text {diff }}(y, \beta, \eta ; \zeta)+\left(3 T_{2}^{a}(\xi, \zeta)-V_{a 1}^{\text {diff }}(\xi, \beta, \eta ; \zeta)\right), \\
T_{5}^{a}(\xi, \zeta) & =\int_{\xi}^{1 / \eta} \frac{d y}{\xi} W_{a 2}^{\text {diff }}(y, \beta, \eta ; \zeta) .
\end{aligned}
$$

The $\beta$ and $\eta$ dependence of $T_{i}^{a}(\xi, \zeta)$ is understood. There are different possibilities to choose the tensor-basis of the hadronic tensor. One way is

$$
\begin{aligned}
\frac{1}{2 \pi} \operatorname{Im} T_{\{\mu \nu\}}(q)= & -g_{\mu \nu}^{\mathrm{T}} \int d \zeta \sum_{a}\left[\frac{q \mathcal{K}_{a}}{q \mathcal{P}} T_{1}^{a}(\xi, \zeta)+\frac{\mathcal{P} \mathcal{K}_{a}}{\mathcal{P}^{2}} T_{2}^{a}(\xi, \zeta)\right] \\
& +\frac{1}{M^{2}} p_{-\mu}^{\mathrm{T}} p_{-\nu}^{\mathrm{T}} \int d \zeta \frac{M^{2}}{\left(\mathcal{P}^{\mathrm{T}}\right)^{2}} \sum_{a}\left[\frac{q \mathcal{K}_{a}}{q \mathcal{P}} T_{3}^{a}(\xi, \zeta)+\frac{\mathcal{P} \mathcal{K}_{a}}{\mathcal{P}^{2}} T_{4}^{a}(\xi, \zeta)\right] \\
+ & \frac{1}{M^{2}}\left(p_{-\mu}^{\mathrm{T}} \pi_{-\nu}^{\mathrm{T}}+p_{-\nu}^{\mathrm{T}} \pi_{-\mu}^{\mathrm{T}}\right) \int d \zeta \zeta \frac{M^{2}}{\left(\mathcal{P}^{\mathrm{T}}\right)^{2}} \sum_{a}\left[\frac{q \mathcal{K}_{a}}{q \mathcal{P}} T_{3}^{a}(\xi, \zeta)+\frac{\mathcal{P} \mathcal{K}_{a}}{\mathcal{P}^{2}} T_{4}^{a}(\xi, \zeta)\right] \\
+ & \frac{1}{M^{2}} \pi_{-\mu}^{\mathrm{T}} \pi_{-\nu}^{\mathrm{T}} \int d \zeta \zeta^{2} \frac{M^{2}}{\left(\mathcal{P}^{\mathrm{T}}\right)^{2}} \sum_{a}\left[\frac{q \mathcal{K}_{a}}{q \mathcal{P}} T_{3}^{a}(\xi, \zeta)+\frac{\mathcal{P} \mathcal{K}_{a}}{\mathcal{P}^{2}} T_{4}^{a}(\xi, \zeta)\right] \\
+ & \frac{1}{M^{2}}\left(p_{-\mu}^{\mathrm{T}} \mathcal{K}_{a \nu}^{\mathrm{T}}+p_{-\nu}^{\mathrm{T}} \mathcal{K}_{a \mu}^{\mathrm{T}}\right) \int d \zeta \frac{M^{2}}{\mathcal{P}^{2}} \sum_{a} T_{5}^{a}(\xi, \zeta) \\
+ & \frac{1}{M^{2}}\left(\pi_{-\mu}^{\mathrm{T}} \mathcal{K}_{a \nu}^{\mathrm{T}}+\pi_{-\nu}^{\mathrm{T}} \mathcal{K}_{a \mu}^{\mathrm{T}}\right) \int d \zeta \zeta \frac{M^{2}}{\mathcal{P}^{2}} \sum_{a} T_{5}^{a}(\xi, \zeta) .
\end{aligned}
$$

Here the two kinematic invariants are $\mathcal{K}^{1 \rho}=p_{-}^{\rho}, \mathcal{K}^{2 \rho}=\pi_{-}^{\rho}$. Introducing the structure functions $U_{i}(\beta, \eta)$ one obtains

$$
\begin{aligned}
\frac{1}{2 \pi} \operatorname{Im} T_{\{\mu \nu\}}(q)= & -g_{\mu \nu}^{\mathrm{T}} U_{1}(\beta, \eta)+\frac{p_{-\mu}^{\mathrm{T}} p_{-\nu}^{\mathrm{T}}}{M^{2}} U_{2}(\beta, \eta) \\
& +\frac{p_{-\mu}^{\mathrm{T}} \pi_{-\nu}^{\mathrm{T}}+p_{-\nu}^{\mathrm{T}} \pi_{-\mu}^{\mathrm{T}}}{M^{2}} U_{3}(\beta, \eta)+\frac{\pi_{-\mu}^{\mathrm{T}} \pi_{-\nu}^{\mathrm{T}}}{M^{2}} U_{4}(\beta, \eta)
\end{aligned}
$$


with

$$
\begin{aligned}
U_{1}(\beta, \eta)= & \int d \zeta\left[T_{1}^{1}(\xi, \zeta)+\frac{\mathcal{P} p_{-}}{\mathcal{P}^{2}} T_{2}^{1}(\xi, \zeta)+\frac{\mathcal{P} \pi_{-}}{\mathcal{P}^{2}} T_{2}^{2}(\xi, \zeta)\right] \\
U_{2}(\beta, \eta)= & \int d \zeta\left\{\frac{M^{2}}{\left(\mathcal{P}^{\mathrm{T}}\right)^{2}}\left[T_{3}^{1}(\xi, \zeta)+\frac{\mathcal{P} p_{-}}{\mathcal{P}^{2}} T_{4}^{1}(\xi, \zeta)+\frac{\mathcal{P} \pi_{-}}{\mathcal{P}^{2}} T_{4}^{2}(\xi, \zeta)\right]+\frac{M^{2}}{\mathcal{P}^{2}} T_{5}^{1}(\xi, \zeta)\right\} \\
U_{3}(\beta, \eta)= & \int d \zeta \zeta\left\{\frac{M^{2}}{\left(\mathcal{P}^{\mathrm{T}}\right)^{2}}\left[T_{3}^{1}(\xi, \zeta)+\frac{\mathcal{P} p_{-}}{\mathcal{P}^{2}} T_{4}^{1}(\xi, \zeta)+\frac{\mathcal{P} \pi_{-}}{\mathcal{P}^{2}} T_{4}^{2}(\xi, \zeta)\right]\right. \\
& \left.+\frac{M^{2}}{\mathcal{P}^{2}}\left[T_{5}^{1}(\xi, \zeta)+\frac{1}{\zeta} T_{5}^{2}(\xi, \zeta)\right]\right\} \\
U_{4}(\beta, \eta)= & \left.\int d \zeta \zeta^{2}\left\{\frac{M^{2}}{\left(\mathcal{P}^{\mathrm{T}}\right)^{2}}\left[T_{3}^{1}(\xi, \zeta)+\frac{\mathcal{P}_{-}}{\mathcal{P}^{2}} T_{4}^{1}(\xi, \zeta)+\frac{\mathcal{P}_{-}}{\mathcal{P}^{2}} T_{4}^{2}(\xi, \zeta)\right]+\frac{M^{2}}{\mathcal{P}^{2}} \frac{1}{\zeta} T_{5}^{2}(\xi, \zeta)\right]\right\} .
\end{aligned}
$$

One may finally change to the basis in (2.10). The unpolarized diffractive structure functions are then obtained by

$$
\begin{aligned}
& W_{1}^{s}(\beta, \eta)=U_{1} \\
& W_{2}^{s}(\beta, \eta)=U_{2}-2\left(1+\frac{1}{\eta}\right) U_{3}+\left(1+\frac{1}{\eta}\right)^{2} U_{4} \\
& W_{3}^{s}(\beta, \eta)=U_{2}+2\left(1-\frac{1}{\eta}\right) U_{3}+\left(1-\frac{1}{\eta}\right)^{2} U_{4} \\
& W_{4}^{s}(\beta, \eta)=-U_{2}+\frac{2}{\eta} U_{3}+\left(1-\frac{1}{\eta^{2}}\right) U_{4},
\end{aligned}
$$

with $U_{i}=U_{i}(\beta, \eta)$. The structure functions $U_{i}$, resp. $W_{i}^{s}$, depend only on the measurable kinematic variables $\beta$ and $\eta$ and form the hadronic tensor (2.10) in the spin-averaged case for general azimuthal angle and can be measured directly.

\section{The Antisymmetric Part of the Amplitude}

The Fourier transform for the antisymmetric part of the amplitude again is taken over from Ref. [27] (cf. Eq. (5.2)):

$$
\begin{array}{r}
T_{[\mu \nu]}^{\mathrm{tw} 2}(q)=\frac{1}{2} \sum_{a} \int D \mathbb{Z} \epsilon^{\mu \alpha \nu \beta} \frac{-q^{2}}{\left[(q \Pi)^{2}-q^{2} \Pi^{2}\right]}\left\{q_{\alpha} \mathcal{K}_{5 \beta}^{a} F_{(5) 1}^{a}(\mathbb{Z})+q_{\alpha} \Pi_{\beta}\left(q \mathcal{K}_{5}^{a}\right) F_{(5) 2}^{a}(\mathbb{Z})\right. \\
\left.+q_{\alpha} \Pi_{\beta}\left(\Pi \mathcal{K}_{5}^{a}\right) F_{(5) 3}^{a}(\mathbb{Z})\right\} \frac{1}{R(1)+\mathrm{i} \epsilon},
\end{array}
$$

with

$$
F_{a 1}^{(5)}(\mathbb{Z})=\left(q \Pi+\Pi^{2}\right) \int_{0}^{1} \frac{d \tau}{\tau^{2}} f_{5 a}\left(\frac{\mathbb{Z}}{\tau}\right)+\Pi^{2} \int_{0}^{1} \frac{d \tau}{\tau^{2}} \int_{0}^{1} \frac{d \tau_{1}}{\tau_{1}^{2}} f_{5 a}\left(\frac{\mathbb{Z}}{\tau \tau_{1}}\right)
$$




$$
\begin{aligned}
F_{a 2}^{(5)}(\mathbb{Z})= & f_{5 a}(\mathbb{Z})-\left(\frac{3 q \Pi\left(q \Pi+\Pi^{2}\right)}{\left[(q \Pi)^{2}-q^{2} \Pi^{2}\right]}-2\right) \int_{0}^{1} \frac{d \tau}{\tau^{2}} f_{5 a}\left(\frac{\mathbb{Z}}{\tau}\right) \\
& -\frac{3(q \Pi) \Pi^{2}}{\left[(q \Pi)^{2}-q^{2} \Pi^{2}\right]} \int_{0}^{1} \frac{d \tau}{\tau^{2}} \int_{0}^{1} \frac{d \tau_{1}}{\tau_{1}^{2}} f_{5 a}\left(\frac{\mathbb{Z}}{\tau \tau_{1}}\right), \\
F_{a 3}^{(5)}(\mathbb{Z})= & f_{5 a}(\mathbb{Z})+\frac{3 q \Pi\left(q^{2}+q \Pi\right)}{\left[(q \Pi)^{2}-q^{2} \Pi^{2}\right]} \int_{0}^{1} \frac{d \tau}{\tau^{2}} f_{5 a}\left(\frac{\mathbb{Z}}{\tau}\right) \\
& +\left(\frac{3(q \Pi)^{2}}{\left[(q \Pi)^{2}-q^{2} \Pi^{2}\right]}-1\right) \int_{0}^{1} \frac{d \tau}{\tau^{2}} \int_{0}^{1} \frac{d \tau_{1}}{\tau_{1}^{2}} f_{5 a}\left(\frac{\mathbb{Z}}{\tau \tau_{1}}\right),
\end{aligned}
$$

Let us remark that the symmetry $(\mathbb{Z}) \rightarrow-(\mathbb{Z})$ in $F_{a i}^{(5)}$ is a consequence of the symmetry in $f_{5 a}$ as has been shown in Ref. [27], App. C. Consequently, all terms $\propto 1 /[R(0)+\mathrm{i} \epsilon]$ drop out.

We consider the imaginary part of this expression. Again, only the terms $\propto 1 /[R(1)+\mathrm{i} \epsilon]$ contribute,

$$
\begin{aligned}
\operatorname{Im} T_{[\mu \nu]}^{\mathrm{tw} 2}(q)= & \frac{\pi}{4} \epsilon_{\mu \nu}{ }^{\alpha \beta} \sum_{a} \int d \zeta \int_{1 / \eta}^{0} \vartheta^{2} \frac{q^{2}}{(\vartheta \kappa)^{3}\left[(q \mathcal{P})^{2}-q^{2} \mathcal{P}^{2}\right]^{3 / 2}} \delta(\vartheta-\xi) \times \\
& \left\{q_{\alpha} \mathcal{K}_{5 \beta}^{a} F_{a 1}^{(5)}(\vartheta, \zeta)+\vartheta \kappa q_{\alpha} \mathcal{P}_{\beta}\left(q \mathcal{K}_{5}^{a}\right) F_{a 2}^{(5)}(\vartheta, \zeta)+(\vartheta \kappa)^{2} q_{\alpha} \mathcal{P}_{\beta}\left(\mathcal{P} \mathcal{K}_{5}^{a}\right) F_{a 3}^{(5)}(\vartheta, \zeta)\right\}
\end{aligned}
$$

It is convenient to introduce the distribution amplitudes $\Phi_{5 a}(i)(\vartheta, \zeta)$

$$
\begin{aligned}
\Phi_{5 a}^{(0)}(\vartheta, \zeta) & \equiv \vartheta f_{5 a}(\vartheta, \zeta) \\
\Phi_{5 a}^{(i)}(\vartheta, \zeta) & =\vartheta \int_{0}^{1} \frac{d \tau_{1}}{\tau_{1}^{2}} \cdots \int_{0}^{1} \frac{d \tau_{i-1}}{\tau_{i-1}^{2}} \int_{0}^{1} \frac{d \tau_{i}}{\tau_{i}^{2}} f_{5 a}\left(\frac{\vartheta}{\tau_{1} \cdots \tau_{i-1} \tau_{i}}, \zeta\right) \\
& =\vartheta \int_{0}^{1} \frac{d \tau_{1}}{\tau_{1}^{2}} \cdots \int_{0}^{1} \frac{d \tau_{i-1}}{\tau_{i-1}^{2}} \int_{\vartheta /\left(\tau_{1} \cdots \tau_{i-1}\right)}^{1 / \eta} \frac{d y_{i}}{y_{i}} \frac{\tau_{1} \cdots \tau_{i-1} y_{i}}{\vartheta} f_{5 a}\left(y_{i}, \zeta\right) \\
& =\int_{\vartheta}^{1 / \eta} \frac{d y_{1}}{y_{1}} \cdots \int_{y_{i-2}}^{1 / \eta} \frac{d y_{i-1}}{y_{i-1}} \int_{y_{i-1}}^{1 / \eta} \frac{d y_{i}}{y_{i}}\left[y_{i} f_{5 a}\left(y_{i}, \zeta\right)\right] \\
& =\int_{\vartheta}^{1 / \eta} \frac{d y}{y} \Phi_{5 a}^{(i-1)}(y, \zeta)
\end{aligned}
$$

where a successive change of variables $y_{m}=\vartheta /\left(\tau_{1} \ldots \tau_{m-1} \tau_{m}\right), m=n, \ldots, 1$, has been made and the support restriction of $f_{5 a}(\vartheta, \zeta)$ to $1 / \eta \leq \vartheta \leq 0$ has been observed.

Then, performing the $\vartheta$-integration with the help of the $\delta$-function, one gets the following expression which simply may be obtained from [27], Eqs. (5.10) replacing there $x \rightarrow-2 \beta$ :

$$
\begin{aligned}
\operatorname{Im} T_{[\mu \nu]}^{\mathrm{tw} 2}(q)= & \frac{\pi}{2} \epsilon_{\mu \nu}{ }^{\alpha \beta} \int d \zeta\left(\frac{4 \beta}{\xi} \frac{1}{\left[1+4 \beta^{2} \mathcal{P}^{2} / Q^{2}\right]^{3 / 2}}\right) \times \\
& \left\{\frac{q_{\alpha} \mathcal{K}_{5 \beta}^{a}}{q \mathcal{P}}\left[\left(1-\beta \xi \mathcal{P}^{2} / Q^{2}\right) \Phi_{5 a}^{(1)}(\xi, \zeta)-\beta \xi \mathcal{P}^{2} / Q^{2} \Phi_{5 a}^{(2)}(\xi, \zeta)\right]\right. \\
& +\frac{q_{\alpha} \mathcal{P}_{\beta}}{q \mathcal{P}} \frac{\left(q \mathcal{K}_{5}^{a}\right)}{q \mathcal{P}}\left[\Phi_{5 a}^{(0)}(\xi, \zeta)-\frac{1+2 \beta \xi \mathcal{P}^{2} / Q^{2}}{\left[1+4 \beta^{2} \mathcal{P}^{2} / Q^{2}\right]^{1 / 2}} \Phi_{5 a}^{(1)}(\xi, \zeta)\right. \\
& \left.+3 \frac{\beta \xi \mathcal{P}^{2} / Q^{2}}{\left[1+4 \beta^{2} \mathcal{P}^{2} / Q^{2}\right]} \Phi_{5 a}^{(2)}(\xi, \zeta)\right]
\end{aligned}
$$




$$
\begin{aligned}
& -\beta \xi \frac{\mathcal{P}^{2}}{Q^{2}} \frac{q_{\alpha}}{q \mathcal{P}} \frac{\left(\mathcal{P} \mathcal{K}_{5}^{a}\right)}{\mathcal{P}^{2}}\left[\Phi_{5 a}^{(0)}(\xi, \zeta)-\frac{3}{\left[1+4 \beta^{2} \mathcal{P}^{2} / Q^{2}\right]^{1 / 2}} \Phi_{5 a}^{(1)}(\xi, \zeta)\right. \\
& \left.\left.+\frac{2-4 \beta^{2} \mathcal{P}^{2} / Q^{2}}{\left[1+4 \beta^{2} \mathcal{P}^{2} / Q^{2}\right]} \Phi_{5 a}^{(2)}(\xi, \zeta)\right]\right\},
\end{aligned}
$$

This equation is written more conveniently as ${ }^{3}$

$$
\begin{gathered}
\operatorname{Im} T_{[\mu \nu]}^{\mathrm{tw} 2}(q)=-\pi \epsilon_{\mu \nu}{ }^{\alpha \beta} \int d \zeta\left\{\frac{q_{\alpha} \mathcal{K}_{5 \beta}^{a}}{q \mathcal{P}}\left[g_{a 1}(\beta, \zeta)+g_{a 2}(\beta, \zeta)\right]-\frac{q_{\alpha} \mathcal{P}_{\beta}}{q \mathcal{P}} \frac{\left(q \mathcal{K}_{5}^{a}\right)}{q \mathcal{P}} g_{2}(\beta, \zeta)\right. \\
\left.+\frac{1}{2} \frac{\mathcal{P}^{2}}{Q^{2}} \frac{q_{\alpha} \mathcal{P}_{\beta}}{q \mathcal{P}} \frac{\left(\mathcal{P} \mathcal{K}_{5}^{a}\right)}{\mathcal{P}^{2}} g_{a 0}(\beta, \zeta)\right\}
\end{gathered}
$$

Here the following equalities

$$
\begin{aligned}
1-\beta \xi \mathcal{P}^{2} / Q^{2} & =\sqrt{1+4 \beta^{2} \mathcal{P}^{2} / Q^{2}}=-(1+4 \beta / \xi) \\
\beta \frac{\partial}{\partial \beta} \xi & =\frac{\xi}{\sqrt{1+4 \beta^{2} \mathcal{P}^{2} / Q^{2}}} \\
\beta \frac{\partial}{\partial \beta} \Phi_{a}^{(i)}(\xi ; \zeta) & =-\frac{\xi}{\sqrt{1+4 \beta^{2} \mathcal{P}^{2} / Q^{2}}} \Phi_{a}^{(i-1)}(\xi ; \zeta) \\
\beta \frac{\partial}{\partial \beta}\left(\frac{2 \beta}{\xi} \frac{1}{\left[1+4 \beta^{2} \mathcal{P}^{2} / Q^{2}\right]^{1 / 2}}\right) & =\frac{2 \beta}{\xi} \frac{1-\left[1+4 \beta^{2} \mathcal{P}^{2} / Q^{2}\right]^{1 / 2}}{\left[1+4 \beta^{2} \mathcal{P}^{2} / Q^{2}\right]^{3 / 2}}=\frac{1}{2} \frac{4 \beta^{2} \mathcal{P}^{2} / Q^{2}}{\left[1+4 \beta^{2} \mathcal{P}^{2} / Q^{2}\right]^{3 / 2}}
\end{aligned}
$$

were used. For the functions $g_{a i}(\beta, \zeta)$ one finds the following expressions:

$$
\begin{aligned}
g_{a 1}(\beta, \zeta) \equiv & -\beta \frac{\partial}{\partial \beta}\left[\beta \frac{\partial}{\partial \beta}\left(\frac{2 \beta}{\xi} \frac{\Phi_{5 a}^{(2)}(\xi, \zeta)}{\left[1+4 \beta^{2} \mathcal{P}^{2} / Q^{2}\right]^{1 / 2}}\right)\right] \\
= & \frac{-2 \beta / \xi}{\left[1+4 \beta^{2} \mathcal{P}^{2} / Q^{2}\right]^{3 / 2}}\left[\Phi_{5 a}^{(0)}(\xi, \zeta)+\frac{2 \beta(2 \beta-\xi) \mathcal{P}^{2} / Q^{2}}{\left[1+4 \beta^{2} \mathcal{P}^{2} / Q^{2}\right]^{1 / 2}} \Phi_{5 a}^{(1)}(\xi, \zeta)\right. \\
& \left.+\beta \xi \mathcal{P}^{2} / Q^{2} \frac{\left(2-4 \beta^{2} \mathcal{P}^{2} / Q^{2}\right)}{\left[1+4 \beta^{2} \mathcal{P}^{2} / Q^{2}\right]} \Phi_{5 a}^{(2)}(\xi, \zeta)\right] \\
g_{a 2}(\beta, \zeta) \equiv & \beta \frac{\partial^{2}}{\partial \beta^{2}} \beta\left(\frac{2 \beta}{\xi} \frac{\Phi_{5 a}^{(2)}(\xi, \zeta)}{\left[1+4 \beta^{2} \mathcal{P}^{2} / Q^{2}\right]^{1 / 2}}\right) \\
= & \frac{2 \beta / \xi}{\left[1+4 \beta^{2} \mathcal{P}^{2} / Q^{2}\right]^{3 / 2}}\left[\Phi_{5 a}^{(0)}(\xi, \zeta)-\frac{1+2 \beta \xi \mathcal{P}^{2} / Q^{2}}{\left[1+4 \beta^{2} \mathcal{P}^{2} / Q^{2}\right]^{1 / 2}} \Phi_{5 a}^{(1)}(\xi, \zeta)\right. \\
& \left.+3 \frac{\beta \xi \mathcal{P}^{2} / Q^{2}}{\left[1+\beta^{2} \mathcal{P}^{2} / Q^{2}\right]} \Phi_{5 a}^{(2)}(\xi, \zeta)\right] \\
g_{a 1}(\beta, \zeta)+g_{a 2}(\beta, \zeta) \equiv & \beta \frac{\partial}{\partial \beta}\left(\frac{2 \beta}{\xi} \frac{\Phi_{5 a}^{(2)}(\xi, \zeta)}{\left[1+4 \beta^{2} \mathcal{P}^{2} / Q^{2}\right]^{1 / 2}}\right) \\
= & \frac{-2 \beta / \xi}{\left[1+x^{2} \mathcal{P}^{2} / Q^{2}\right]^{3 / 2}}\left[\left(1-\beta \xi \mathcal{P}^{2} / Q^{2}\right) \Phi_{5 a}^{(1)}(\xi, \zeta)-\beta \xi \mathcal{P}^{2} / Q^{2} \Phi_{5 a}^{(2)}(\xi, \zeta)\right],
\end{aligned}
$$

\footnotetext{
${ }^{3}$ The implicit $\eta$-dependence has been suppressed for brevity.
} 


$$
\begin{aligned}
g_{a 0}(\beta, \zeta)= & \beta^{2} \frac{\partial^{2}}{\partial \beta^{2}}\left(4 \beta^{2} \frac{\Phi_{5 a}^{(2)}(\xi, \zeta)}{\left[1+4 \beta^{2} \mathcal{P}^{2} / Q^{2}\right]^{1 / 2}}\right) \\
= & \frac{4 \beta^{2}}{\left[1+4 \beta^{2} \mathcal{P}^{2} / Q^{2}\right]^{3 / 2}}\left[\Phi_{5 a}^{(0)}(\xi, \zeta)-\frac{3}{\left[1+4 \beta^{2} \mathcal{P}^{2} / Q^{2}\right]^{1 / 2}} \Phi_{5 a}^{(1)}(\xi, \zeta)\right. \\
& \left.+\frac{2-4 \beta^{2} \mathcal{P}^{2} / Q^{2}}{\left[1+4 \beta^{2} \mathcal{P}^{2} / Q^{2}\right]} \Phi_{5 a}^{(2)}(\xi, \zeta)\right] .
\end{aligned}
$$

Obviously, the contributions to the various kinematic expressions are related to only one function. Namely, introducing

$$
\mathcal{F}_{a}(\beta, \eta, \zeta)=\frac{-2 \beta}{\xi} \frac{\Phi_{5 a}^{(2)}(\xi, \zeta)}{\left[1+4 \beta^{2} \mathcal{P}^{2} / Q^{2}\right]^{1 / 2}}
$$

we may rewrite the functions $g_{a i}$ as follows

$$
\begin{aligned}
g_{a 1}(\beta, \eta, \zeta) & =\beta \frac{\partial}{\partial \beta} \beta \frac{\partial}{\partial \beta} \mathcal{F}_{a}(\beta, \eta, \zeta) \\
g_{a 2}(\beta, \eta, \zeta) & =-\beta \frac{\partial}{\partial \beta}\left(\beta \frac{\partial}{\partial \beta}+1\right) \mathcal{F}_{a}(\beta, \eta, \zeta) \\
g_{a 0}(\beta, \eta, \zeta) & =-2 \beta \frac{\partial}{\partial \beta}\left(\beta \frac{\partial}{\partial \beta}-1\right) \beta \xi \mathcal{F}_{a}(\beta, \eta, \zeta) .
\end{aligned}
$$

One finally has to perform the $\zeta$-integral to obtain the diffractive structure functions in the polarized case. The distribution functions $\left.g_{a i}(\beta, \eta, \zeta)\right|_{i=0} ^{2}$ occur in products with $\left.\zeta^{k}\right|_{k=0} ^{2}$ and result into six structure functions of the form for each invariant $a$

$$
\begin{aligned}
G_{a j}(\beta, \eta) & =\int d \zeta g_{a j}(\beta, \eta, \zeta), \\
H_{a j}(\beta, \eta) & =\int d \zeta \zeta g_{a j}(\beta, \eta, \zeta), \\
K_{a j}(\beta, \eta) & =\int d \zeta \zeta^{2} g_{a j}(\beta, \eta, \zeta),
\end{aligned}
$$

leading to

$$
\begin{aligned}
\operatorname{Im} T_{[\mu \nu]}^{\mathrm{tw} 2}(q)=-\pi \epsilon_{\mu \nu}{ }^{\alpha \beta} & \left\{\frac{q_{\alpha} \mathcal{K}_{5 \beta}^{a}}{q p_{-}}\left(G_{a 1}(\beta, \eta)+G_{a 2}(\beta, \eta)\right)\right. \\
& -\frac{q_{\alpha} p_{-\beta}}{q p_{-}}\left(\frac{q \mathcal{K}_{5}^{a}}{q p_{-}} G_{a 2}(\beta, \eta)-\frac{1}{2} \frac{p_{-} \mathcal{K}_{5}^{a}}{Q^{2}} G_{a 0}(\beta, \eta)-\frac{1}{2} \frac{\pi_{-} \mathcal{K}_{5}^{a}}{Q^{2}} H_{a 0}(\beta, \eta)\right) \\
& \left.-\frac{q_{\alpha} \pi_{-\beta}}{q p_{-}}\left(\frac{q \mathcal{K}_{5}^{a}}{q p_{-}} H_{a 2}(\beta, \eta)-\frac{1}{2} \frac{p_{-} \mathcal{K}_{5}^{a}}{Q^{2}} H_{a 0}(\beta, \eta)-\frac{1}{2} \frac{\pi_{-} \mathcal{K}_{5}^{a}}{Q^{2}} K_{a 0}(\beta, \eta)\right)\right\} .
\end{aligned}
$$


Inserting the three invariants $\mathcal{K}_{a 5}$ explicitly one obtains

$$
\begin{aligned}
\operatorname{Im} T_{[\mu \nu]}^{\mathrm{tw} 2}(q) & =-\pi \epsilon_{\mu \nu}^{\alpha \beta}\left\{\frac{q_{\alpha} S_{\beta}^{\mathrm{T}}}{q p_{-}}\left(G_{11}+G_{12}\right)\right. \\
+ & \frac{q_{\alpha} p_{-\beta}^{\mathrm{T}}}{q p_{-}}\left[-\frac{q S}{q p_{-}} G_{12}+\frac{p_{2} S}{M^{2}}\left[G_{21}\right.\right. \\
& \left.\left.\left.+\frac{1}{2 Q^{2}}\left(p_{-}^{2} G_{20}+p_{-} \pi_{-}\left(G_{30}+H_{20}\right)+\pi_{-}^{2} H_{30}\right)+M^{2} G_{10}+M^{2} \frac{\eta-1}{\eta} H_{10}\right)\right]\right] \\
+ & \frac{q_{\alpha} \pi_{-\beta}^{\mathrm{T}}}{q p_{-}}\left[-\frac{q S}{q p_{-}} H_{12}(\beta, \eta)+\frac{p_{2} S}{M^{2}}\left[G_{31}+G_{32}-H_{22}\right.\right. \\
& \left.\left.\left.+\frac{1}{2 Q^{2}}\left(p_{-}^{2} H_{20}+p_{-} \pi_{-}\left(H_{30}+K_{20}\right)+\pi_{-}^{2} K_{30}+M^{2} H_{10}+M^{2} \frac{\eta-1}{\eta} K_{10}\right)\right]\right]\right\},
\end{aligned}
$$

with

$$
p_{-}^{2}=t, \quad p_{-} \pi_{-}=-t / \eta, \quad \pi_{-}^{2}=4 M^{2}-t\left(1-1 / \eta^{2}\right), \quad \frac{\eta-1}{\eta}=\frac{2 \beta}{x} .
$$

This structure of the hadronic tensor specifies the general structure obtained for three characteristic 4 -vectors $p_{1}, p_{2}$ and $S$ in Eq. (2.13) for the present process. One may cast (4.23) into the form of (2.13) using Shouten identities [33]:

$$
\begin{aligned}
& X_{\mu} \varepsilon_{\nu \rho \sigma \tau}=X_{\nu} \varepsilon_{\mu \rho \sigma \tau}+X_{\rho} \varepsilon_{\nu \mu \sigma \tau}+X_{\sigma} \varepsilon_{\nu \rho \mu \tau}+X_{\tau} \varepsilon_{\nu \rho \sigma \mu} \\
& g_{\lambda \mu} \varepsilon_{\nu \rho \sigma \tau}=g_{\lambda \nu} \varepsilon_{\mu \rho \sigma \tau}+g_{\lambda \rho} \varepsilon_{\nu \mu \sigma \tau}+g_{\lambda \sigma} \varepsilon_{\nu \rho \mu \tau}+g_{\lambda \tau} \varepsilon_{\nu \rho \sigma \mu} .
\end{aligned}
$$

Note that the structure functions $J_{k 0}, \quad J=G, H, K$ are suppressed by $\mu^{2} / Q^{2}$ relative to the other contributions, with $\mu^{2}$ a hadronic scale. In the Bjorken limit, these structure functions do not contribute.

\section{$5 \quad$ Relations between Diffractive Structure Functions}

Relations between different structure functions as observed in deep-inelastic scattering [12,21-23, 25] hold also for polarized and unpolarized diffractive scattering in the limit $M^{2}, t \rightarrow 0$, cf. $[7,8]$. The situation is more involved for the case studied in the present paper, since the structure functions emerge as a $\zeta$-integral of sub-system structure functions, which accounts for the twoparticle nature of the wave-function. Thus the corresponding relations can be established for the un-integrated $\zeta$-dependent functions only.

\subsection{Unpolarized Case}

As before in the case of deep-inelastic scattering [11,12] and non-forward scattering [27] we seek for a representation of the distribution functions $W_{1,2, L}^{\mathrm{diff}}, V_{a(0,1)}^{\mathrm{diff}}$ in terms of a single function. One may choose the distribution functions $\Phi_{a}^{(2)}(\xi ; \zeta)\left(\right.$ (3.10) and express the functions $W_{1,2, L}^{\text {diff }}, V_{a(0,1)}^{\text {diff }}$ 
through the following differential relations :

$$
\begin{aligned}
W_{a \mathrm{~L}}^{\text {diff }}(\xi(\beta), \beta, \eta ; \zeta) & =\frac{\mathcal{P}^{2}}{Q^{2}} \beta^{2} \frac{\partial}{\partial \beta}\left(\frac{-2 \beta}{\xi(\beta)} \frac{\Phi_{a}^{(2)}(\xi(\beta) ; \zeta)}{\sqrt{1+4 \beta^{2} \mathcal{P}^{2} / Q^{2}}}\right), \\
\frac{2 q \mathcal{P}}{\mathcal{P}^{2}} W_{a 2}^{\text {diff }}(\xi(\beta), \beta, \eta ; \zeta) & =\beta^{2} \frac{\partial^{2}}{\partial \beta^{2}}\left(\frac{4 \beta^{2}}{\xi(\beta)^{2}} \frac{\Phi_{a}^{(2)}(\xi(\beta) ; \zeta)}{\sqrt{1+4 \beta^{2} \mathcal{P}^{2} / Q^{2}}}\right),
\end{aligned}
$$

Note that the longitudinal distribution function $W_{a \mathrm{~L}}^{\operatorname{diff}}(\xi(\beta), \beta, \eta ; \zeta)$ vanishes in the limit $\mathcal{P}^{2} / Q^{2} \rightarrow 0$.

A generalized Callan-Gross relation, which holds for diffractive scattering in the limit $M^{2}, t \rightarrow 0,[7]$, is broken as in the case of deep-inelastic scattering [11]. The distribution functions $W_{(1,2)}^{\text {diff }}$ are related by

$$
\begin{aligned}
W_{a 1}^{\text {diff }}(\xi(\beta), \beta, \eta ; \zeta)+W_{a \mathrm{~L}}^{\operatorname{diff}}(\xi(\beta), \beta, \eta ; \zeta) & =\frac{\left(1+4 \beta^{2} \mathcal{P}^{2} / Q^{2}\right)}{(-4 \beta)} \frac{2 q \mathcal{P}}{\mathcal{P}^{2}} W_{a 2}^{\text {diff }}(\xi(\beta), \beta, \eta ; \zeta) \\
& =\frac{\left(\mathcal{P}^{\mathrm{T}}\right)^{2}}{\mathcal{P}^{2}} W_{a 2}^{\operatorname{diff}}(\xi(\beta), \beta, \eta ; \zeta) .
\end{aligned}
$$

The distribution functions $V_{a(0,1)}^{\text {diff }}$ can be expressed by the distribution functions $W_{1, L}^{\text {diffr }}$ directly

$$
\begin{aligned}
2 V_{a 0}^{\text {diff }}(\xi(\beta), \beta, \eta ; \zeta)= & W_{a L}^{\text {diff }}(\xi(\beta), \beta, \eta ; \zeta)-2 W_{a 1}^{\text {diff }}(\xi(\beta), \beta, \eta ; \zeta), \\
\frac{1}{2} V_{a 1}^{\text {diff }}(\xi(\beta), \beta, \eta ; \zeta)= & \sqrt{1+4 \beta^{2} \mathcal{P}^{2} / Q^{2}} \beta \frac{\partial}{\partial \beta} W_{a L}^{\text {diff }}(\xi(\beta), \beta, \eta ; \zeta) \\
& +\left(1-\frac{2}{\sqrt{1+4 \beta^{2} \mathcal{P}^{2} / Q^{2}}}\right) W_{a L}^{\operatorname{diff}}(\xi(\beta), \beta, \eta ; \zeta) \\
& -\frac{4 \beta^{2} \mathcal{P}^{2} / Q^{2}}{\left[1+4 \beta^{2} \mathcal{P}^{2} / Q^{2}\right]^{3 / 2}} \int_{\beta}^{1} \frac{d \rho}{\rho^{2}} W_{a L}^{\text {diff }}(\xi(\beta \rho), \beta \rho, \eta ; \zeta) .
\end{aligned}
$$

Three of the above distribution functions are independent. To obtain the four diffractive structure functions on the level of observables $\left.W_{i}^{s}\right|_{i=1} ^{4}$ in the unpolarized case (2.10) the $\zeta$-integral has to be performed. As shown in section 3 the respective linear combinations are weighted by different $\zeta$-dependent functions, that in general no relations exist on the level of structure functions.

\subsection{Polarized Case}

Below the $\zeta$-integral the distribution functions $\left.g_{a i}\right|_{i=0} ^{2}$ are all functions of $\mathcal{F}_{a}(\beta, \eta, \zeta)$ (4.18). Therefore one may express the functions $g_{a 0}$ and $g_{a 2}$ in terms of $g_{a 1}$ as the central function. This is achieved applying the relations

$$
\begin{aligned}
\beta \frac{\partial}{\partial \beta} \mathcal{F}_{a}(\beta, \eta, \zeta) & =-\int_{\beta}^{1} \frac{d y}{y} g_{a 1}^{\mathrm{tw} 2}(y, \eta, \zeta) \\
\mathcal{F}_{a}(\beta, \eta, \zeta) & =-\int_{\beta}^{1} \frac{d y}{y} \int_{y}^{1} \frac{d y^{\prime}}{y^{\prime}} g_{a 1}^{\mathrm{tw} 2}\left(y^{\prime}, \eta, \zeta\right) .
\end{aligned}
$$

We consider twist-2 operators only and find the Wandzura-Wilczek relation

$$
g_{a 2}^{\mathrm{tw} 2}(\beta, \eta, \zeta)=-g_{a 1}^{\mathrm{tw} 2}(\beta, \eta, \zeta)+\int_{\beta}^{1} \frac{d y}{y} g_{a 1}^{\mathrm{tw} 2}(y, \eta, \zeta)
$$


between $g_{a 2}^{\mathrm{tw} 2}$ and $g_{a 1}^{\mathrm{tw} 2}$. All target mass and $t$-corrections are absorbed into the structure functions. Note that this relation holds for all invariants $a$ independently. The validity of the Wandzura-Wilczek relation also in case of diffractive scattering at general hadronic scales $M^{2}, t$ is a further example in a long list of cases: covariant parton model [23,24,34], target- and quarkmass corrections [12,14] gluon-induced heavy flavor production [35], non-forward scattering [25], diffractive scattering in the limit $M^{2}, t \rightarrow 0$ [8].

The distribution function $g_{a 0}^{\mathrm{tw} 2}$ obeys the relation

$$
\begin{aligned}
g_{a 0}^{\mathrm{tw} 2}(\beta, \eta, \zeta)= & -2 \beta \xi g_{a 1}^{\mathrm{tw} 2}(\beta, \eta, \zeta)-\frac{8 \beta^{2}-2 \beta \xi}{\left[1+4 \beta^{2} \mathcal{P}^{2} / Q^{2}\right]^{1 / 2}} \int_{\beta}^{1} \frac{d y}{y} g_{a 1}^{\mathrm{tw} 2}(y, \eta, \zeta) \\
& -\frac{8 \beta^{2}}{\left[1+4 \beta^{2} \mathcal{P}^{2} / Q^{2}\right]^{3 / 2}} \int_{\beta}^{1} \frac{d y}{y} \int_{y}^{1} \frac{d y^{\prime}}{y^{\prime}} g_{a 1}^{\mathrm{tw} 2}\left(y^{\prime}, \eta, \zeta\right) .
\end{aligned}
$$

It contributes to parts of the hadronic tensor which are suppressed by an overall factor $\mu^{2} / Q^{2}$, with $\mu$ a hadronic mass scale. In the related case of non-forward scattering the emergence of this function has been observed in Ref. [25].

For $g_{a 2}$ the $\zeta$-integral can be performed maintaining the structure of relation (5.8). Therefore the Wandzura-Wilczek relation also holds for the diffractive structure functions

$$
\begin{aligned}
& G_{a 2}^{\mathrm{tw} 2}(\beta, \eta)=-G_{a 1}^{\mathrm{tw} 2}(\beta, \eta)+\int_{\beta}^{1} \frac{d y}{y} G_{a 1}^{\mathrm{tw} 2}(y, \eta) \\
& H_{a 2}^{\mathrm{tw} 2}(\beta, \eta)=-H_{a 1}^{\mathrm{tw} 2}(\beta, \eta)+\int_{\beta}^{1} \frac{d y}{y} H_{a 1}^{\mathrm{tw} 2}(y, \eta) .
\end{aligned}
$$

Due to the $\zeta$-dependence of $g_{a 0}$ through $\xi$ and $\mathcal{P}^{2}$ one needs to know $g_{a 1}(\beta, \eta, \zeta)$ to calculate the structure functions $J_{a, 0}(\beta, \eta)$. As the integral (4.20) cannot be inverted, relations between the structure functions $J_{a, 0}$ and the other polarized structure functions cannot be established unless referring to the un-integrated distribution functions $g_{a 1}(\beta, \eta, \zeta)$.

\section{Conclusions}

Deep-inelastic diffractive scattering, like other hard scattering processes off nucleons, requires target mass corrections in the region of lower scales of $Q^{2}$. In fact the nucleon mass $M$ is not the only hadronic scale relevant to this process in which both the incoming and outgoing nucleon play a role. The modulus of the invariant $t=\left(p_{2}-p_{1}\right)^{2}$ on average is of the same size as $M^{2} .{ }^{4}$ It appears to be natural to seek for a formalism dealing with both the target mass and $t$-effects simultaneously. Diffractive factorization and the use of A. Mueller's generalized optical theorem made it possible to reformulate diffractive scattering in terms of deep-inelastic scattering off an effective two-nucleon state accounting for $t$. We applied the formalism of the (non-local) light-cone expansion in the case of non-forward scattering and adapted it to the kinematics present in deep-inelastic diffractive scattering. The formalism accounting for target mass and $t$-corrections was then implemented generalizing the picture derived in the limit $M^{2}, t \rightarrow 0$ in earlier work both for the unpolarized and polarized case. The present formalism can in principle be extended to the case of higher twist concerning the corresponding operator expressions $\Omega_{\alpha}^{(5)}$. However, besides factorizable contributions also non-factorizable terms have to be described.

\footnotetext{
${ }^{4}$ In case of related semi-exclusive processes in which more than one final-state hadron is well separated in rapidity from the inclusively produced hadrons other invariants more would emerge.
} 
While in the limit $M^{2}, t \rightarrow 0$ all diffractive structure functions can be expressed in terms of diffractive parton densities, this is no longer the case at lower scales of $Q^{2}$ in regions where $M^{2}$ and $t$-corrections become relevant. Here we mean the kinematic $t$ contributions which have to be distinguished from the dynamic contributions emerging in the non-perturbative distribution functions. The reason for this is that the 2-particle kinematics of the incoming and outgoing nucleon deviates from quasi-collinearity which was instrumental to unify the boost variables of both particles to a single one. The condition for the absorptive part is now related to a $\delta$-distribution with a more complicated argument leaving the integral over the relative transverse motion of the two nucleons. This integral cannot be inverted in general referring to the accessible kinematic variables. The corresponding distribution functions $\Phi_{a(5)}(\xi, \beta, \eta, \zeta ; t)$ have to be determined by non-perturbative methods in the future.

Although the partonic picture does not hold for the diffractive structure functions one still finds it below the $\zeta$-integral. At the level of twist-2 the structure functions can be built from the corresponding operator expectation values as in the case of deep-inelastic scattering since the specifics of diffractive scattering is moved into the corresponding two-particle wave-functions. Consequently, the logarithmic scaling violations, which can be completely associated with that of the operators, cf. $[7,17]$, are found to be the same as in diffractive scattering changing the variables $x$ in the deep-inelastic case to $\beta$ in the diffractive case.

The presence of target mass and $t$-effects enlarges the number of structure functions determining the hadronic tensor. In the unpolarized case four structure functions contribute, which cannot be related to each other directly. The Callan-Gross relation does not hold. However, in the polarized case the Wandzura-Wilzcek relation remains unbroken and holds even separately for the contributions to the different invariants $\left.\mathcal{K}_{a}\right|_{a=3} ^{5}$. In addition to the structure functions surviving in the limit $M^{2}, t \rightarrow 0, Q^{2} \rightarrow \infty$ several new structure functions contribute. They are damped however $\propto 1 / Q^{2}$ w.r.t. the other contributions. The present formalism can be used in experimental analysis of deep-inelastic diffractive scattering data referring to suitable models for the un-integrated distribution functions depending on $\zeta$, for which rigorous determination using methods of non-perturbative QCD do not yet exits. In this way the structures being derived in the present paper can be tested.

Acknowledgments: This work was supported in part by DFG Sonderforschungsbereich Transregio 9, Computergestützte Theoretische Physik. 


\section{References}

[1] M. Derrick et al. [ZEUS Collaboration], Phys. Lett. B 315 (1993) 481;

T. Ahmed et al. [H1 Collaboration], Nucl. Phys. B 429 (1994) 477.

[2] M. Derrick et al. [ZEUS Collaboration], Z. Phys. C 70 (1996) 391 [arXiv:hep-ex/9602010;

C. Adloff et al. [H1 Collaboration], Z. Phys. C 76 (1997) 613 [arXiv/hep-ex/9708016;

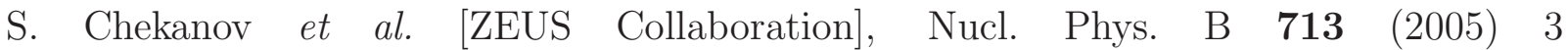
[arXiv:hep-ex/0501060].

[3] C. Adloff et al. [H1 Collaboration], Phys. Lett. B 393 (1997) 452 [arXiv:hep-ex/9611017.

[4] H. Abramowicz and J. B. Dainton, J. Phys. G 22 (1996) 911.

[5] G. Ingelman and P. E. Schlein, Phys. Lett. B 152 (1985) 256;

J. Bartels and G. Ingelman, Phys. Lett. B 235 (1990) 175;

A. Donnachie and P. V. Landshoff, Phys. Lett. B 518 (2001) 63 [arXiv:hep-ph/0105088;

Phys. Lett. B 191 (1987) 309 [Erratum-ibid. B 198 (1987) 590]; Phys. Lett. B 437 (1998) 408 [arXiv hep-ph/9806344]; Nucl. Phys. B 244 (1984) 322; references quoted therein;

W. Buchmüller and A. Hebecker, Phys. Lett. B 355 (1995) 573 [arXiv:hep-ph/9504374;

M. Wüsthoff and A. D. Martin, J. Phys. G 25 (1999) R309 [arXiv hep-ph/9909362;

A. Hebecker, Phys. Rep. 331 (2000) 1

J. Bartels, J. R. Ellis, H. Kowalski and M. Wüsthoff, Eur. Phys. J. C 7 (1999) 443 [arXiv hep-ph/9803497;

J. Bartels and H. Kowalski, Eur. Phys. J. C 19 (2001) 693 [arXiv hep-ph/0010345;

H. Navelet and R. Peschanski, [arXiv:hep-ph/0105030] and references quoted therein;

G. Ingelman, [arXiv hep-ph/9912534 and references therein;

M. Diehl, T. Feldmann, R. Jakob, and P. Kroll, Phys. Lett. B460 (1999) 204; Eur. Phys.

J. C8 (1999) 409;

P. Kroll, Nucl. Phys. A666 (2000) 3.

[6] A. Berera and D. E. Soper, Phys. Rev. D 53 (1996) 6162 [arXiv/hep-ph/9509239];

A. Berera, arXiv hep-ph/9606448.

J. C. Collins, Phys. Rev. D 57 (1998) 3051 [Erratum-ibid. D 61 (2000) 019902] [arXiv hep-ph/9709499.

[7] J. Blümlein and D. Robaschik, Phys. Lett. B 517 (2001) 222 [arXiv/hep-ph/0106037.

[8] J. Blümlein and D. Robaschik, Phys. Rev. D 65 (2002) 096002 [arXivihep-ph/0202077].

[9] J. Blumlein and D. Robaschik, Nucl. Phys. A 711 (2002) 228 [arXiv/hep-ph/0207250].

[10] O. Nachtmann, Nucl. Phys. B 63 (1973) 237.

[11] H. Georgi and H. D. Politzer, Phys. Rev. D 14 (1976) 1829.

[12] J. Blümlein and A. Tkabladze, Nucl. Phys. B 553 (1999) 427 [arXiv hep-ph/9812478.

[13] J. Blümlein and A. Tkabladze, Nucl. Phys. Proc. Suppl. 79 (1999) 541 [arXiv:hep-ph/9905524].

[14] A. Piccione and G. Ridolfi, Nucl. Phys. B 513 (1998) 301 [arXiv hep-ph/9707478]. 
[15] V. Baluni and E. Eichten, Phys. Rev. D 14 (1976) 3045; Phys. Rev. Lett. 37 (1976) 1181;

S. Wandzura, Nucl. Phys. B 122 (1977) 412;

S. Matsuda and T. Uematsu, Nucl. Phys. B 168 (1980) 181;

R. L. Jaffe and M. Soldate, Phys. Rev. D 26 (1982) 49;

H. Kawamura and T. Uematsu, Phys. Lett. B 343 (1995) 346 [arXiv hep-ph/9409455.

[16] S.A. Anikin and O.I. Zavialov, Ann. Phys. (N.Y.) 116 (1978) 135,

M. Bordag and D. Robaschik, Nucl. Phys. B169 (1980) 445,

M. Bordag, B. Geyer, J. Hořejši, and D. Robaschik, Z. Phys. C26 (1985) 275,

O.I. Zavialov, Renormalized Quantum Field Theory, Dordrecht 1990,

O.I. Zavialov, Theor. Math. Phys. 138 (2004) 370, [translated from: Teor. Mat. Fiz. 138 (2004) 437].

[17] J. Blümlein, B. Geyer and D. Robaschik, Nucl. Phys. B 560 (1999) 283 [arXiv:hep-ph/9903520].

[18] B. Geyer, M. Lazar and D. Robaschik, Nucl. Phys. B 559 (1999) 339 [arXiv; hep-th/9901090;

B. Geyer and M. Lazar, Nucl. Phys. B 581 (2000) 341 [arXiv:hep-th/0003080.

[19] J. Eilers, B. Geyer and M. Lazar, Phys. Rev. D 69 (2004) 034015.

[20] J. Eilers, The decomposition of local and nonlocal operators with repect to irreducible representations of the orthogonal group and some of its applications in Quantum Chromodynamics, PhD Thesis, Leipzig 2004;

J. Eilers, Geometric twist decomposition off the light-cone for non-local QCD operators, to appear.

[21] C. G. Callan and D. J. Gross, Phys. Rev. Lett. 22 (1969) 156.

[22] S. Wandzura and F. Wilczek, Phys. Lett. B 72 (1977) 195.

[23] J. Blümlein and N. Kochelev, Nucl. Phys. B 498 (1997) 285 [arXiv hep-ph/9612318.

[24] J. Blümlein and N. Kochelev, Phys. Lett. B 381 (1996) 296 [arXiv:hep-ph/9603397.

[25] J. Blümlein and D. Robaschik, Nucl. Phys. B 581 (2000) 449 [arXiv:hep-ph/0002071].

[26] B. Geyer and D. Robaschik, Phys. Rev. D 71 (2005) 054018 [arXiv hep-ph/0407301.

[27] B. Geyer, D. Robaschik and J. Eilers, Nucl. Phys. B 704 (2005) 279 [arXiv:hep-ph/0407300.

[28] cf. S. Capitani, Acta Phys.Polon. B33 (2002) 3025;

M. Guagnelli et al., Eur. Phys. J. A17 (2003) 365;

M. Göckeler, R. Horsley, D. Pleiter, P. E. L. Rakow, A. Schäfer, G. Schierholz and W. Schroers, [QCDSF Collaboration], Phys. Rev. Lett. 92 (2004) 042002 [arXiv:hep-ph/0304249.

P. Hägler, J. Negele, D. B. Renner, W. Schroers, T. Lippert and K. Schilling, [LHPC collaboration], Phys. Rev. D 68 (2003) 034505 [arXiv:hep-lat/0304018;

M. J. Savage, Talk given at 23rd International Symposium on Lattice Field Field: Lattice 2005, Trinity College, Dublin, Ireland, 25-30 Jul 2005, PoS LAT2005:020, 2006, hep-lat/0509048;

S. R. Beane, P. F. Bedaque, K. Orginos and M. J. Savage, arXiv hep-lat/0602010. 
[29] A. H. Mueller, Phys. Rev. D 2 (1970) 2963; Phys. Rev. D 4 (1971) 150;

P.D.P. Collins, An Introduction to Regge Theory and High Energy Physics, Cambridge 1977, pp. 331;

A. A. Logunov, B. V. Medvedev, M. A. Mestvirishvili, V. P. Pavlov, M. K. Polivanov and A. D. Sukhanov, Theor. Math. Phys. 33 (1978) 935 [Teor. Mat. Fiz. 33 (1977) 149].

[30] J. Blümlein, J. Eilers, B. Geyer and D. Robaschik, Phys. Rev. D 65 (2002) 054029 [arXiv hep-ph/0108095].

[31] B. Geyer, M. Lazar and D. Robaschik, Nucl. Phys. B 618 (2001) 99 [Erratum-ibid. B 652 (2003) 408] [arXiv hep-ph/0108061.

[32] A. V. Belitsky and D. Müller, Phys. Lett. B 507 (2001) 173 [arXiv/hep-ph/0102224.

[33] M. J. G. Veltman, Nucl. Phys. B 319 (1989) 253;

B. De Wit and J. Smith, Field Theory in Particle Physics, (North Holland, Amsterdam, 1986), p. 414.

[34] R. G. Roberts and G. G. Ross, Phys. Lett. B 373 (1996) 235 [arXiv hep-ph/9601235].

[35] J. Blümlein, V. Ravindran and W. L. van Neerven, Phys. Rev. D 68 (2003) 114004 [arXiv:hep-ph/0304292]. 